\title{
Impact of Thermal Non-equilibrium on Weak Nonlinear Rotating Porous Convection
}

\author{
R. N. Dayananda ${ }^{1} \cdot$ I. S. Shivakumara ${ }^{1}$
}

Received: 6 May 2019 / Accepted: 19 September 2019 / Published online: 4 October 2019

(c) Springer Nature B.V. 2019

\begin{abstract}
The consequences of local thermal non-equilibrium (LTNE) on both stationary and oscillatory weak nonlinear stability of gravity-driven porous convection in an incompressible fluid-saturated rotating porous layer are investigated. A stability map is drawn in the DarcyTaylor and scaled Vadasz number plane to demarcate the regions of stationary and oscillatory convection, and thereby, co-dimension- 2 points are determined. It is found that the effect of increasing interphase heat transfer coefficient is to enhance the region of stationary convection and decrease the region of oscillatory convection. The complex Ginzburg-Landau equations are derived using the multi-scale method, and pitchfork and Hopf bifurcations occur at stationary and oscillatory critical Darcy-Rayleigh numbers, respectively. The linear and nonlinear oscillatory neutral curves are illustrated, and at the quartic point, the transition from supercritical to subcritical bifurcations is identified for the governing parameters. The impact of LTNE model is to enhance the region of forward bifurcation and post-transient amplitude compared to LTE case. Heat transfer is obtained in terms of Nusselt number for both stationary and oscillatory convection. The region of enhancement in heat flux for oscillatory convection in the smaller scaled Vadasz number domain with increasing Darcy-Taylor number increases with increasing interphase heat transfer coefficient and the porosity-modified conductivity ratio.
\end{abstract}

Keywords Nonlinear convection · Local thermal non-equilibrium · Porous medium • Rotation

\section{List of Symbols}

a Horizontal wave number

c Specific heat

Da Darcy number, $K / d^{2}$

R. N. Dayananda

dayanandarn@gmail.com

I. S. Shivakumara

shivakumarais@bub.ernet.in

1 Department of Mathematics, Bangalore University, Bengaluru 560056, India 
$d \quad$ Depth of the porous layer

$\vec{g} \quad$ Gravitational acceleration

$H \quad$ Non-dimensional interphase heat transfer coefficient, $h d^{2} / \varepsilon k_{\mathrm{f}}$

$h \quad$ Interphase heat transfer coefficient

$K \quad$ Permeability of the porous medium

$k \quad$ Thermal conductivity

$\operatorname{Pr} \quad$ Prandtl number, $v / \kappa_{\mathrm{f}}$

$R_{\mathrm{TD}} \quad$ Darcy-Rayleigh number, $\beta_{\mathrm{T}} g\left(T_{\mathrm{L}}-T_{\mathrm{U}}\right) K d / \nu \kappa_{\mathrm{f}} \varepsilon$

$T \quad$ Temperature

Ta Darcy-Taylor number, $(2 \Omega K / \nu \varepsilon)^{2}$

$t \quad$ Time

$V a \quad$ Vadasz number, $\operatorname{Pr} \varepsilon / D a$

$x, y, z$ Space coordinates

\section{Greek Symbols}

$\alpha \quad$ Ratio of diffusivities, $\kappa_{\mathrm{f}} / \kappa_{\mathrm{S}}$

$\beta_{\mathrm{T}}$ Thermal expansion coefficient

$\delta \quad$ Scaled Vadasz number, $V a / \pi^{2}$

$\varepsilon \quad$ Porosity

$\gamma$ Porosity-modified conductivity ratio, $\varepsilon k_{\mathrm{f}} /(1-\varepsilon) k_{\mathrm{S}}$

$\kappa_{\mathrm{f}} \quad$ Thermal diffusivity of the fluid, $k_{\mathrm{f}} /(\rho C)_{\mathrm{f}}$

$\kappa_{\mathrm{S}} \quad$ Thermal diffusivity of the solid, $k_{\mathrm{s}} /(\rho C)_{\mathrm{s}}$

$\mu \quad$ Dynamic viscosity of the fluid

$v$ Kinematic viscosity of the fluid, $\left(\mu / \rho_{0}\right)$

$\vec{\Omega}$ Angular velocity $(0,0, \Omega)$

$\omega$ Frequency

$\psi \quad$ Stream function

\section{Introduction}

Buoyancy-driven convection in a fluid-saturated porous medium with a local thermal nonequilibrium (LTNE) model where the fluid temperature differs from the solid temperature is an interesting topic of research and studied extensively (Banu and Rees 2002; Malashetty et al. 2005a, b; Rees and Pop 2005; Straughan 2006, 2010; Rees 2010; Lee et al. 2011; Saravanan and Sivakumar 2011; Barletta and Rees 2012; Straughan 2013; Celli et al. 2013; Barletta and Rees 2015). The gargantuan literature existing on this topic is well documented in the books by Nield and Bejan (2017) and Straughan (2015). The reasons for the increased attention of LTNE effects are numerous applications in real life. For example, there are applications in tube refrigerators in space, in fuel cells, in processing composite materials, in nuclear reactor maintenance, in heat exchangers, in biological tissue analysis, in flow and heat transfer in porous metallic foams and in convection in stellar atmospheres (for details see Straughan 2013 and references therein).

Thermal convective instability in a fluid-saturated porous medium under the rotational effects is in itself an interesting topic, and by adopting a local thermal equilibrium (LTE) model, the problem has been studied extensively (Palm and Tyvand 1984; Vadasz 1993a, b, 
1994, 1996, 1998; Straughan 2001; Vadasz and Govender 2001; Govender 2003; Malashetty et al. 2007; Saravanan 2009; Falsaperla et al. 2010; Capone and Rionero 2013). In his seminal paper, Vadasz (1998) compared the results of thermal convection in rotating porous media with the analogous results of non-porous domain and unveiled the existence of overstable solutions without limiting the scaled Vadasz number. The importance of the paper by Straughan (2001) lies in performing a global nonlinear stability of rotating porous convection and the inclusion of a time derivative term in the extended Darcy equation, a practice motivated by Vadasz (1998). Furthermore, the importance of a dimensionless group $(\varepsilon \operatorname{Pr} / \mathrm{Da}$, where $\varepsilon$ is the porosity of the porous medium, $P r$ is the Prandtl number and $D a$ is the Darcy number) was realized by Vadasz (1998) in his study and Straughan (2001) named the group as the Vadasz number. The Vadasz number is found to be of order unity for some modern porous media applications such as fractured porous media, and hence, the acceleration term cannot be ignored in those situations.

The analysis of LTNE effects on rotating porous convection is of recent origin. Straughan (2006) was the first to investigate the effect of rotation on thermal convection in a porous medium using a LTNE model and demonstrated the equivalence of the linear instability and nonlinear stability boundaries through the energy approach. Both thermal and mechanical anisotropies of the porous medium on the onset of convection in a rotating porous layer are discussed by Govender and Vadasz (2007), under LTNE effects. Malashetty et al. (2007) discussed linear and weak nonlinear stability analyses of thermal convection in a rotating fluid-saturated porous layer considering LTNE model, while Malashetty and Swamy (2007) and (2010) extended this problem to consider the effects of anisotropy and sparseness of the porous medium using the Brinkman-extended Darcy equation, respectively. Thermal convective instability in a layer of Darcy porous medium subject to alternating direction of centrifugal body force, when the layer fails to exhibit thermal equilibrium, is dealt by Saravanan and Brindha (2013), whereas Capone and Gentile (2018) studied the effect of a uniform rotation on linear and nonlinear thermal convection in an anisotropic porous medium with LTNE model by ignoring the time derivative in the velocity.

Nonetheless, it is intriguing to examine the effect of LTNE on nonlinear steady and oscillatory rotating Darcy-Bénard convection and to bring out anomalous results, if any, compared to LTE model, by including the time derivative in the velocity. In the present study, the analysis of Vadasz (1998) is extended to carry out this task. The stability of bifurcating equilibrium solution is studied by deriving the Ginzburg-Landau equations for both steady and oscillatory motions. Following Rees (2009), the nonlinear oscillatory solution curves are compared with the linear oscillatory ones to know the existence of subcritical/supercritical bifurcation and thereby quartic points (i.e. where the nonlinear oscillatory neutral curve branches off the linear curve) are located for considered governing parameters. Besides, heat transfer for both stationary and oscillatory convection is analysed in terms of Nusselt number.

\section{Governing Equations}

We consider an incompressible Boussinesq fluid-saturated horizontal layer of Darcy porous medium of thickness $d$. A rectangular Cartesian coordinate system $(x, y, z)$ is chosen such that the origin is at the bottom of the porous layer and the $z$-axis is measured vertically upward. The gravity is acting in the negative $z$-direction. The porous layer rotates uniformly about the $z$-axis with constant angular velocity $\vec{\Omega}$ and also assumed to be in LTNE. The fluid temperature $T_{\mathrm{f}}$ and the solid temperature $T_{\mathrm{s}}$ are maintained at constant temperatures 
$T_{\mathrm{L}}$ and $T_{\mathrm{U}}$ at the lower and upper boundaries, respectively. The flow in the porous medium is described by the modified Darcy law including the time derivative and the Coriolis force. The basic equations are:

$$
\begin{gathered}
\nabla \cdot \vec{q}=0, \\
\frac{\rho_{0}}{\varepsilon} \frac{\partial \vec{q}}{\partial t}=-\nabla p+\rho_{0}\left[1-\beta_{\mathrm{T}}\left(T_{\mathrm{f}}-T_{\mathrm{L}}\right)\right] \vec{g}-\frac{2}{\varepsilon}(\vec{\Omega} \times \vec{q})-\frac{\mu_{\mathrm{f}}}{K} \vec{q}, \\
\varepsilon(\rho c)_{\mathrm{f}} \frac{\partial T_{\mathrm{f}}}{\partial t}+(\rho c)_{\mathrm{f}}(\vec{q} \cdot \nabla) T_{\mathrm{f}}=\varepsilon k_{\mathrm{f}} \nabla^{2} T_{\mathrm{f}}+h\left(T_{\mathrm{s}}-T_{\mathrm{f}}\right), \\
(1-\varepsilon)(\rho c)_{\mathrm{s}} \frac{\partial T_{\mathrm{s}}}{\partial t}=(1-\varepsilon) k_{\mathrm{s}} \nabla^{2} T_{\mathrm{s}}-h\left(T_{\mathrm{s}}-T_{\mathrm{f}}\right),
\end{gathered}
$$

where $\vec{q}=(u, v, w)$ is the velocity vector, $\vec{g}$ is the gravitational acceleration, $p$ is the pressure, $\mu_{\mathrm{f}}$ is the fluid viscosity, $K$ is the permeability of the porous medium, $\varepsilon$ is the porosity of the porous medium, $c$ is the specific heat, $h$ is the interphase heat transfer coefficient, $k_{\mathrm{f}}$ and $k_{\mathrm{S}}$ are the thermal conductivities of fluid phase and solid phase, respectively, $\beta_{\mathrm{T}}$ is the coefficient of thermal expansion and $\rho_{0}$ is the reference density.

The above equations are subject to the following boundary conditions:

$$
\vec{q} \cdot \hat{k}=0 \text { at } z=0, d, \quad T_{\mathrm{f}}=T_{\mathrm{S}}=T_{\mathrm{L}} \text { at } z=0, \quad T_{\mathrm{f}}=T_{\mathrm{s}}=T_{\mathrm{U}} \text { at } z=d .
$$

The basic state is quiescent and given by

$$
\begin{aligned}
\vec{q}_{\mathrm{b}} & =0, p_{\mathrm{b}}(z)=p_{0}-\rho_{0} g z-\rho_{0} \beta_{\mathrm{t}} g\left(T_{\mathrm{L}}-T_{\mathrm{U}}\right) z^{2} / 2 d, \\
T_{\mathrm{fb}}(z) & =T_{\mathrm{L}}-\left(T_{\mathrm{L}}-T_{\mathrm{U}}\right) z / d=T_{\mathrm{sb}}(z) .
\end{aligned}
$$

The basic state is perturbed in the form

$$
\vec{q}=\vec{q}_{\mathrm{b}}+\vec{q}^{\prime}, \quad p=p_{\mathrm{b}}(z)+p^{\prime}, \quad T_{\mathrm{f}}=T_{\mathrm{fb}}(z)+T_{\mathrm{f}}^{\prime}, \quad T_{\mathrm{s}}=T_{\mathrm{sb}}(z)+T_{\mathrm{s}}^{\prime},
$$

where the primes denote the perturbations over their equilibrium counterparts. Substituting Eq. (7) into Eqs. (1)-(4), using Eq. (6), eliminating the pressure term from the momentum equation by operating curl once, defining a two-dimensional stream function $\psi^{\prime}(x, z, t)$ in the form

$$
u^{\prime}=\partial \psi^{\prime} / \partial z, \quad w^{\prime}=-\partial \psi^{\prime} / \partial x,
$$

and using $d, k_{\mathrm{f}} /(\rho c)_{\mathrm{f}} d$ and $T_{\mathrm{L}}-T_{\mathrm{U}}$ to scale length, velocity and temperature, respectively, we obtain finally the governing stability equations in the non-dimensional form as follows (after neglecting the primes):

$$
\begin{gathered}
{\left[\left(\frac{\partial}{\partial \tau}+1\right)^{2} \nabla^{2}+T a \frac{\partial^{2}}{\partial z^{2}}\right] \psi=-R_{\mathrm{TD}}\left(\frac{\partial}{\partial \tau}+1\right) \frac{\partial T_{\mathrm{f}}}{\partial x}} \\
\left(V a \frac{\partial}{\partial \tau}-\nabla^{2}+H\right) T_{\mathrm{f}}-H T_{\mathrm{s}}=-\frac{\partial \psi}{\partial x}+J\left(\psi, T_{\mathrm{f}}\right), \\
\left(\alpha V a \frac{\partial}{\partial \tau}-\nabla^{2}+\gamma H\right) T_{\mathrm{s}}-\gamma H T_{\mathrm{f}}=0 .
\end{gathered}
$$

Here, $V a=\nu \varepsilon d^{2} / \kappa_{\mathrm{f}} K$ is the Vadasz number, $R_{\mathrm{TD}}=\beta_{\mathrm{T}} g\left(T_{\mathrm{L}}-T_{\mathrm{U}}\right) K d / \nu \kappa_{\mathrm{f}} \varepsilon$ is the Darcy-Rayleigh number, $T a=(2 \Omega K / \nu \varepsilon)^{2}$ is the Darcy-Taylor number, $\alpha=\kappa_{\mathrm{f}} / \kappa_{\mathrm{S}}$ is the ratio of diffusivities of fluid phase to solid phase, $\gamma=\varepsilon k_{\mathrm{f}} /(1-\varepsilon) k_{\mathrm{s}}$ is the porosity-modified 
conductivity ratio of fluid phase to solid phase, $H=h d^{2} / \varepsilon k_{\mathrm{f}}$ is the non-dimensional interphase heat transfer coefficient, $\tau=(V a) t, J(. .$, ..) stands for the Jacobian with respect to $x$ and $z$ and $\nabla^{2}=\partial^{2} / \partial x^{2}+\partial^{2} / \partial z^{2}$.

The boundary conditions are:

$$
\psi=T_{\mathrm{f}}=T_{\mathrm{s}}=0 \text { at } z=0,1 .
$$

\section{Weak Nonlinear Stability Analysis}

To perform the weak nonlinear stationary and oscillatory stability analyses, multi-scale method adopted by Vadasz (1998) is used. The Ginzburg-Landau equations are derived and the stability of bifurcating equilibrium solution is analysed. Moreover, heat transfer is calculated in terms of Nusselt number.

\subsection{Stationary Convection}

To continue with the analysis, it is advantageous to write the governing equations in the operator form

$$
L_{1} \vec{\zeta}=0, \quad L_{2} \vec{\zeta}=J\left(\psi, T_{\mathrm{f}}\right), \quad L_{3} \vec{\zeta}=0
$$

where

$$
\begin{aligned}
& L_{1}=\left[\left(\frac{\partial}{\partial \tau}+1\right)^{2} \nabla^{2}+T a \frac{\partial^{2}}{\partial z^{2}}, R_{\mathrm{TD}}\left(\frac{\partial}{\partial \tau}+1\right) \frac{\partial}{\partial x}, 0\right], \\
& L_{2}=\left[\frac{\partial}{\partial x}, \chi \frac{\partial}{\partial \tau}-\nabla^{2}+H,-H\right], \\
& L_{3}=\left[0,-\gamma H, \alpha \chi \frac{\partial}{\partial \tau}-\nabla^{2}+\gamma H\right], \\
& \vec{\zeta}=\left[\psi, T_{\mathrm{f}}, T_{\mathrm{s}}\right]^{T} .
\end{aligned}
$$

The dependent variables are expanded as a series involving different powers of a small parameter $\phi$ in the form

$$
\left[\psi, T_{\mathrm{f}}, T_{\mathrm{s}}\right]=\phi\left[\psi^{(1)}, T_{\mathrm{f}}^{(1)}, T_{\mathrm{s}}^{(1)}\right]+\phi^{2}\left[\psi^{(2)}, T_{\mathrm{f}}^{(2)}, T_{\mathrm{s}}^{(2)}\right]+\phi^{3}\left[\psi^{(3)}, T_{\mathrm{f}}^{(3)}, T_{\mathrm{s}}^{(3)}\right]+\cdots,
$$

where $\phi=\left(R_{\mathrm{TD}} / R_{\mathrm{TD}_{\mathrm{cr}}}-1\right)^{1 / 2}$ and it provides the expression for the Darcy-Rayleigh number. A slow timescale $\tau=\phi^{2} t^{\prime}$ is introduced to avoid exponential growth rate and to get finite amplitude solutions, and also, a slow space scale is introduced in the form $X=\phi x$, to obtain finite bandwidth of modes. Substituting Eq. (14) as well as slow time and space scales into Eq. (13) and equating terms containing powers of $\phi$ provide a system of linear partial differential equations at every order.

The following system of linear partial differential equations is obtained at the first order in $\phi$ :

$$
L_{1}^{(\mathrm{St})} \vec{\zeta}_{1}=0, \quad L_{2}^{(\mathrm{St})} \vec{\zeta}_{1}=0, \quad L_{3}^{(\mathrm{St})} \vec{\zeta}_{1}=0
$$

where

$$
\vec{\zeta}_{1}=\left[\psi^{(1)}, T_{\mathrm{f}}^{(1)}, T_{\mathrm{s}}^{(1)}\right]^{T}
$$




$$
\begin{array}{r}
L_{1}^{(\mathrm{St})}=\left[\nabla^{2}+T a \frac{\partial^{2}}{\partial z^{2}}, R_{\mathrm{TD}} \frac{\partial}{\partial x}, 0\right], \\
L_{2}^{(\mathrm{St})}=\left[\frac{\partial}{\partial x},-\nabla^{2}+H,-H\right], \\
L_{3}^{(\mathrm{St})}=\left[0,-\gamma H,-\nabla^{2}+\gamma H\right] .
\end{array}
$$

Eigenparameters of the steady convection corresponding to linear instability theory determine the first-order solution (i.e. the solution of Eq. 15) in the form

$$
\begin{aligned}
& \psi^{(1)}=\left(A_{1} e^{i a x}+A_{1}^{*} e^{-i a x}\right) \sin \pi z, \\
& T_{\mathrm{f}}^{(1)}=\left(B_{1} e^{i a x}+B_{1}^{*} e^{-i a x}\right) \sin \pi z, \\
& T_{\mathrm{s}}^{(1)}=\left(C_{1} e^{i a x}+C_{1}^{*} e^{-i a x}\right) \sin \pi z .
\end{aligned}
$$

The eigenvalue for the steady convection is found to be

$$
R^{(\mathrm{St})}=\left[\frac{H_{1}}{\left(H_{1} \gamma+m+1\right)}+1\right]\left[\frac{(m+1)^{2}}{m}+\frac{\operatorname{Ta}(m+1)}{m}\right],
$$

where $m=a^{2} / \pi^{2}, R=R_{\mathrm{TD}} / \pi^{2}, \delta=V a / \pi^{2}$ and $H_{1}=H / \pi^{2}$. Equation (17) coincides with Malashetty et al. (2007) and with Vadasz (1998) when $H_{1}=0$.

Amplitudes at the first order are related in the form

$$
\begin{aligned}
& B_{1}=\frac{-i m^{1 / 2}\left(p^{0}+H_{1} \gamma\right) A_{1}}{\pi p^{0}\left(p^{0}+H_{1}+H_{1} \gamma\right)}, \quad C_{1}=\frac{-i m^{1 / 2} H_{1} \gamma A_{1}}{\pi p^{0}\left(p^{0}+H_{1}+H_{1} \gamma\right)}, \\
& B_{1}^{*}=\frac{-i m^{1 / 2}\left(p^{0}+H_{1} \gamma\right) A_{1}^{*}}{\pi p^{0}\left(p^{0}+H_{1}+H_{1} \gamma\right)}, \quad C_{1}^{*}=\frac{-i m^{1 / 2} H_{1} \gamma A_{1}^{*}}{\pi p^{0}\left(p^{0}+H_{1}+H_{1} \gamma\right)},
\end{aligned}
$$

where $p^{0}=1+m$. The undetermined amplitudes $A_{1}$ and $A_{1}^{*}$ are determined by using the solvability condition on the third-order system of linear partial differential equations.

The system of equations at the second order in $\phi$ is written in the form

$$
L_{1}^{(\mathrm{St})} \vec{\zeta}_{2}=G_{21}, \quad L_{2}^{(\mathrm{St})} \vec{\zeta}_{2}=G_{22}, \quad L_{3}^{(\mathrm{St})} \vec{\zeta}_{2}=G_{23}
$$

where

$$
\begin{aligned}
& \vec{\zeta}_{2}=\left[\psi^{(2)}, T_{\mathrm{f}}^{(2)}, T_{\mathrm{s}}^{(2)}\right]^{T}, \quad G_{21}=-2 \frac{\partial}{\partial X} \frac{\partial \psi^{(1)}}{\partial x}-R_{\mathrm{TD}_{\mathrm{cr}}} \frac{\partial T_{\mathrm{f}}^{(1)}}{\partial X} \\
& G_{22}=-2 \frac{\partial}{\partial X} \frac{\partial T_{\mathrm{f}}^{(1)}}{\partial x}+\frac{\partial \psi^{(1)}}{\partial X}-\frac{\partial \psi^{(1)}}{\partial x} \frac{\partial T_{\mathrm{f}}^{(1)}}{\partial z}+\frac{\partial \psi^{(1)}}{\partial z} \frac{\partial T_{\mathrm{f}}^{(1)}}{\partial x}, \quad G_{23}=-2 \frac{\partial}{\partial X} \frac{\partial T_{\mathrm{s}}^{(1)}}{\partial x} .
\end{aligned}
$$

The terms $G_{21}, G_{22}$ and $G_{23}$ stand for the inhomogeneous part, which are evaluated by using the known solutions obtained at the first order in $\phi$. The complete solution at the second order contains two parts, namely the complementary function and the particular integral. The complementary function resembles the solution obtained at the first order, and the particular solution is calculated by decoupling the equations (i.e. to get a single equation). 
The complete solution at the second order in $\phi$ is obtained as follows:

$$
\begin{aligned}
& \psi^{(2)}=\left(A_{2} e^{i a x}+A_{2}^{*} e^{-i a x}\right) \sin \pi z, \\
& T_{\mathrm{f}}^{(2)}=\left(B_{2} e^{i a x}+B_{2}^{*} e^{-i a x}\right) \sin \pi z-\frac{m\left(p^{0}+H_{1} \gamma\right)\left(4+\gamma H_{1}\right)}{2 \pi\left(4+H_{1}(1+\gamma)\right) p^{0}\left(p^{0}+H_{1}+H_{1} \gamma\right)} A_{1} A_{1}^{*} \sin 2 \pi z, \\
& T_{\mathrm{s}}^{(2)}=\left(C_{2} e^{i a x}+C_{2}^{*} e^{-i a x}\right) \sin \pi z-\frac{m \gamma H_{1}\left(p^{0}+H_{1} \gamma\right)}{2 \pi\left(4+H_{1}(1+\gamma)\right) p^{0}\left(p^{0}+H_{1}+H_{1} \gamma\right)} A_{1} A_{1}^{*} \sin 2 \pi z .
\end{aligned}
$$

The amplitudes at the second order are related as similar to Eq. (18), i.e. as similar to the relationship between the first-order amplitudes.

The system of equations at the third order in $\phi$ is

$$
L_{1}^{(\mathrm{St})} \vec{\zeta}_{3}=G_{31}, \quad L_{2}^{(\mathrm{St})} \vec{\zeta}_{3}=G_{32}, \quad L_{3}^{(\mathrm{St})} \vec{\zeta}_{3}=G_{33}
$$

where

$$
\begin{aligned}
\vec{\zeta}_{3}= & {\left[\psi^{(3)}, T_{\mathrm{f}}^{(3)}, T_{\mathrm{s}}^{(3)}\right]^{T}, } \\
G_{31} & =-2 \frac{\partial}{\partial X} \frac{\partial \psi^{(2)}}{\partial x}-\frac{\partial^{2} \psi^{(1)}}{\partial X^{2}}-2 \frac{\partial}{\partial \tau}\left(\nabla^{2} \psi^{(1)}\right)-R_{\mathrm{TD}_{\mathrm{cr}}} \frac{\partial}{\partial \tau}\left(\frac{\partial T_{\mathrm{f}}^{(1)}}{\partial x}\right) \\
& -R_{\mathrm{TD}_{\mathrm{cr}}} \frac{\partial T_{\mathrm{f}}^{(1)}}{\partial x}-R_{\mathrm{TD}_{\mathrm{cr}}} \frac{\partial T_{\mathrm{f}}^{(2)}}{\partial X}, \\
G_{32}= & \chi \frac{\partial T_{\mathrm{f}}^{(1)}}{\partial \tau}-2 \frac{\partial}{\partial X} \frac{\partial T_{\mathrm{f}}^{(2)}}{\partial x}-\frac{\partial^{2} T_{\mathrm{f}}^{(1)}}{\partial X^{2}}+\frac{\partial \psi^{(2)}}{\partial X}-\frac{\partial \psi^{(1)}}{\partial x} \frac{\partial T_{\mathrm{f}}^{(2)}}{\partial z}+\frac{\partial \psi^{(1)}}{\partial z} \frac{\partial T_{\mathrm{f}}^{(2)}}{\partial x} \\
& -\frac{\partial \psi^{(2)}}{\partial x} \frac{\partial T_{\mathrm{f}}^{(1)}}{\partial z}+\frac{\partial \psi^{(2)}}{\partial z} \frac{\partial T_{\mathrm{f}}^{(1)}}{\partial x}-\frac{\partial \psi^{(1)}}{\partial X} \frac{\partial T_{\mathrm{f}}^{(1)}}{\partial z}+\frac{\partial \psi^{(1)}}{\partial z} \frac{\partial T_{\mathrm{f}}^{(1)}}{\partial X}, \\
G_{33} & =-2 \frac{\partial}{\partial X} \frac{\partial T_{\mathrm{s}}^{(2)}}{\partial x}-\frac{\partial^{2} T_{\mathrm{s}}^{(1)}}{\partial X^{2}}+\alpha \chi \frac{\partial T_{\mathrm{s}}^{(1)}}{\partial \tau} .
\end{aligned}
$$

The inhomogeneous terms $G_{31}, G_{32}$ and $G_{33}$ include the solutions obtained at the previous orders. The equations at the third order in $\phi$ are the inhomogeneous version of equations at the first order in $\phi$. This enables us to find the first-order amplitude solutions. To derive the solvability condition, first we reduce the linear system at the third order in $\phi$ into a single equation (decoupling) and evaluate the inhomogeneous terms by using the known solutions obtained at the previous orders. The relevant inhomogeneous terms to obtain the solvability condition will include the eigenfunctions corresponding to the homogeneous operator with homogeneous boundary conditions. The resulting differential equation obtained by decoupling is made solvable by equating the coefficients of $e^{i a x} \sin \pi z$ and $e^{-i a x} \sin \pi z$ to zero in relevant inhomogeneous terms. Hence, the solvability condition is obtained as a partial differential equation in the form

$$
Q_{1} \frac{\partial A}{\partial t}-Q_{2} \frac{\partial^{2} A}{\partial x^{2}}=\frac{\pi^{2} m^{2}}{2} Q_{3}\left(\xi_{\mathrm{St}}^{0}-A A^{*}\right) A,
$$

where

$$
\begin{aligned}
& A=\phi A_{1}, A^{*}=\phi A_{1}^{*}, \\
& Q_{1}=\frac{1}{\delta}\left\{m R_{\mathrm{cr}}^{(\mathrm{St})}\left\{H_{1}^{2} m \delta \gamma-\left(p^{0}+H_{1} \gamma\right) p^{0}\left(p^{0}+H_{1}(1+\gamma)\right)\right\}\right.
\end{aligned}
$$




$$
\begin{aligned}
& \left.+m \delta R_{\mathrm{cr}}^{(\mathrm{St})}\left(p^{0}+H_{1} \gamma\right)^{2}+2 p^{0^{3}}\left(p^{0}+H_{1}(1+\gamma)\right)^{2}\right\}, \\
& Q_{2}=m R_{\mathrm{cr}}^{(\mathrm{St})} H_{1}^{2} \gamma+\left(p^{0}+H_{1}(1+\gamma)\right)^{2} p^{0^{2}}+m R_{\mathrm{cr}}^{(\mathrm{St})}\left(p^{0}+H_{1} \gamma\right)^{2}, \\
& Q_{3}=\frac{R_{\mathrm{cr}}^{(\mathrm{St})}\left(p^{0}+H_{1} \gamma\right)^{2}\left(4+H_{1} \gamma\right)}{\left(4+H_{1}(1+\gamma)\right)}, \\
& \xi_{0}^{(\mathrm{St})}=\frac{2 p^{0}\left(p^{0}+H_{1}(1+\gamma)\right)\left(p^{0}+H_{1} \gamma\right)\left(4+H_{1}(1+\gamma)\right)\left(R-R_{\mathrm{cr}}^{(\mathrm{St})}\right)}{m R_{\mathrm{cr}}^{(\mathrm{St})}\left(p^{0}+H_{1} \gamma\right)^{2}\left(4+H_{1} \gamma\right)} .
\end{aligned}
$$

Equation (22) is known as the Ginzburg-Landau equation for the steady case in which original time and space scales have been reintroduced. When $H_{1}=0$, Eq. (22) reduces to

$$
\frac{\left[p^{0}(2-m)+m \delta\right]}{\delta} \frac{\partial A}{\partial t}-p^{0} \frac{\partial^{2} A}{\partial x^{2}}=\frac{\pi^{2} m^{2}}{2}\left[\frac{2 p^{0}}{m}\left(\frac{R}{R_{\mathrm{cr}}^{(\mathrm{St})}}-1\right)-A A^{*}\right] A
$$

and coincides with the one obtained by Vadasz (1998). In the Ginzburg-Landau equation, the diffusion term will appear due to the introduction of slow space scale. By neglecting the diffusion term in Eq. (22), we arrive at the following ordinary differential equation:

$$
Q_{1} \frac{\mathrm{d} E}{\mathrm{~d} t}=\frac{\pi^{2} m^{2}}{8} Q_{3}\left(\xi_{\mathrm{St}}-E^{2}\right) E
$$

where $E=2 i A$ and $\xi_{\mathrm{St}}=4 \xi_{\mathrm{St}}^{0}$. Equation (24) provides steady-state amplitude solution in the form

$$
E=\left\{\begin{array}{lr}
0 & \forall R<R_{\mathrm{cr}}^{(\mathrm{St})} \\
\pm\left(\xi_{\mathrm{St}}\right)^{1 / 2} & \forall R \geq R_{\mathrm{cr}}^{(\mathrm{St})}
\end{array}\right.
$$

From Eq. (25), it is observed that the pitchfork bifurcation occurs at $R_{\mathrm{cr}}^{(\mathrm{St})}$. (i.e. the local bifurcation in which the transition of the system from one fixed point to three fixed points occurs).

The relaxation time $Q_{1}>0$ when $\delta>\delta_{\mathrm{t}}^{(\mathrm{St})}$ and negative when $\delta<\delta_{\mathrm{t}}^{(\mathrm{St})}$, where

$$
\delta_{t}^{(\mathrm{St})}=\frac{\left(1+m_{\mathrm{cr}}^{(\mathrm{St})}\right)\left(1+m_{\mathrm{cr}}^{(\mathrm{St})}+H_{1} \gamma\right)\left\{1+m_{\mathrm{cr}}^{(\mathrm{St})}+H_{1}(1+\gamma)\right\}\left\{T a-\left(1+m_{\mathrm{cr}}^{(\mathrm{St})}\right)\right\}}{\left(T a+1+m_{\mathrm{cr}}^{(\mathrm{St})}\right)\left\{H_{1}^{2} \alpha \gamma+\left(1+m_{\mathrm{cr}}^{(\mathrm{St})}+H_{1} \gamma\right)^{2}\right\}},
$$

and note that $\delta_{t}^{(\mathrm{St})} \geq 0$ provided $T a \geq\left(1+m_{\mathrm{cr}}^{(\mathrm{St})}\right)$.

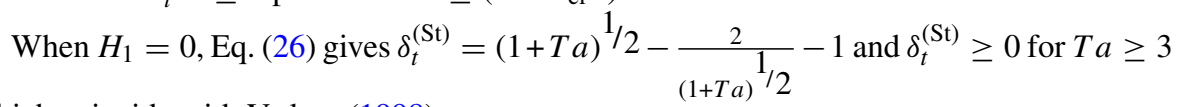
which coincide with Vadasz (1998).

The heat transfer is obtained in terms of thermal Nusselt number. The average Nusselt number for stationary convection is defined as

$$
\overline{N u}_{\mathrm{f}}(\mathrm{St})=\frac{1}{\left(2 \pi / a_{\mathrm{cr}}\right)}\left[\int_{0}^{\left(2 \pi / a_{\mathrm{cr}}\right)}\left(-\partial T_{\mathrm{f}} / \partial z\right) \mathrm{d} x\right]_{z=0} .
$$


Substituting the steady-state amplitude solution corresponding to stationary convection in the absence of slow space scale yields the average Nusselt number for the stationary convection in the form

$$
\overline{N u}_{\mathrm{f}}^{(\mathrm{St})}=1+2\left(\frac{R}{R_{\mathrm{cr}}^{(\mathrm{St})}}-1\right)
$$

\subsection{Oscillatory Convection}

The solution procedure is similar to that of the previous case. Here, two slow timescales are allowed in the form $\tau=\phi^{2} t^{\prime}, \tau_{0}=\phi t^{\prime}$ and again the short time $t^{\prime}$ is rescaled as $\tilde{t}=\omega_{0} t^{\prime}$ where $\omega_{0}=\omega_{\text {cr }}$ (Vadasz 1998). Amplitude fluctuations are studied by using the short time $t^{\prime}$. Substituting Eq. (14) with slow timescales expansions and slow space scale $X=\phi x$ into Eq. (13) provides the linear system of partial differential equations at $o(\phi)$ in the form

$$
L_{1}^{(\mathrm{Osc})} \vec{\zeta}_{1}=0, \quad L_{2}^{(\mathrm{Osc})} \vec{\zeta}_{1}=0, \quad L_{3}^{(\mathrm{Osc})} \vec{\zeta}_{1}=0
$$

where

$$
\begin{aligned}
& L_{1}^{(\mathrm{Osc})}=\left[\left(\omega_{0} \frac{\partial}{\partial \tilde{t}}+1\right)^{2} \nabla^{2}+T a \frac{\partial^{2}}{\partial z^{2}}, R_{\mathrm{TD}_{\mathrm{cr}}}\left(\omega_{0} \frac{\partial}{\partial \tilde{t}}+1\right) \frac{\partial}{\partial x}, 0\right], \\
& L_{2}^{(\mathrm{Osc})}=\left[\frac{\partial}{\partial x}, \chi \omega_{0} \frac{\partial}{\partial \tilde{t}}-\nabla^{2}+H,-H\right], \\
& L_{3}^{(\mathrm{Osc})}=\left[0,-\gamma H, \alpha \chi \omega_{0} \frac{\partial}{\partial \tilde{t}}-\nabla^{2}+\gamma H\right], \\
& \vec{\zeta}_{1}=\left[\psi^{(1)}, T_{\mathrm{f}}^{(1)}, T_{\mathrm{s}}^{(1)}\right]^{T}, \text { and } \psi^{(1)} \text { is written as } \\
& \psi^{(1)}=\left(A_{1} e^{i(a x+\tilde{t})}+B_{1} e^{i(a x-\tilde{t})}+A_{1}^{*} e^{-i(a x+\tilde{t})}+B_{1}^{*} e^{-i(a x-\tilde{t})}\right) \sin \pi z,
\end{aligned}
$$

which stands for a travelling wave solution by the amplitudes $A_{1}\left(\tau_{0}, \tau, X\right)$ and $B_{1}\left(\tau_{0}, \tau, X\right)$. It is seen that $B_{1}=0$ stands for pure left travelling wave, $A_{1}=0$ represents pure right travelling wave and the standing waves (i.e. velocity becomes zero) are categorized by $A_{1}=$ $\pm B_{1}$ or $A_{1}= \pm B_{1}^{*}$. Introducing the symmetry about the axis of rotation at the origin $(x=0)$ provides the special type of standing waves $\left(\psi^{(1)}=0\right)$ categorized by $B_{1}^{*}=-A_{1}$ and $B_{1}=$ $-A_{1}^{*}$; thereby, travelling waves are ignored. By removing the slow space scale, we can avoid the existence of diffusion term in the Ginzburg-Landau amplitude equation. Introducing symmetry conditions at the origin and ignoring the slow space scale yield the following solution at the first order in $\phi$ that balances the linear theory corresponding to oscillatory convection:

$$
\begin{aligned}
{\left[\psi^{(1)}, T_{f}^{(1)}, T_{s}^{(1)}\right]=} & 2\left[i\left(A_{1} e^{i \tilde{t}}-A_{1}^{*} e^{-i \tilde{t}}\right) \sin a x,\left(B_{1} e^{i \tilde{t}}+B_{1}^{*} e^{-i \tilde{t}}\right) \cos a x,\right. \\
& \left.\left(C_{1} e^{i \tilde{t}}+C_{1}^{*} e^{-i \tilde{t}}\right) \cos a x\right] \sin \pi z .
\end{aligned}
$$

The eigenvalue for the oscillatory convection is

$$
R^{(\mathrm{Osc})}=\frac{(1+m)\left(1+m-\delta \omega^{2}\right)}{m}+\frac{(1+m) H_{1}\left\{(1+m)\left(1+m+H_{1} \gamma\right)+\alpha \delta\left(-H_{1} \gamma+\alpha \delta\right) \omega^{2}\right\}}{m\left\{\left(1+m+H_{1} \gamma\right)^{2}+\alpha^{2} \delta^{2} \omega^{2}\right\}}
$$




$$
+\frac{T a\left(1+m+\delta \omega^{2}\right)}{m\left(1+\omega^{2}\right)}+\frac{H_{1} T a\left\{(1+m)\left(1+m+H_{1} \gamma\right)+\alpha \delta\left(H_{1} \gamma+\alpha \delta\right) \omega^{2}\right\}}{m\left(1+\omega^{2}\right)\left\{\left(1+m+H_{1} \gamma\right)^{2}+\alpha^{2} \delta^{2} \omega^{2}\right\}},
$$

where $\omega^{2}(>0)$ is computed from the equation

$$
c_{0}\left(\omega^{2}\right)^{2}+c_{1}\left(\omega^{2}\right)+c_{2}=0 .
$$

Here,

$$
\begin{aligned}
c_{0}= & p^{0} \alpha^{2} \delta^{2}\left(p^{0}+H_{1}+\delta\right), \\
c_{1}= & p^{0^{2}}\left(p^{0}+H_{1} \gamma\right)\left(p^{0}+H_{1}+H_{1} \gamma\right)+p^{0}\left(p^{0^{2}}+H_{1} \gamma\left(2 p^{0}+H_{1}(\alpha+\gamma)\right)\right) \delta \\
& +\left(p^{0}+H_{1}\right)\left(p^{0}-T a\right) \alpha^{2} \delta^{2}+\left(p^{0}+T a\right) \alpha^{2} \delta^{3}, \\
c_{2}= & p^{0}\left(p^{0}-T a\right)\left(p^{0}+H_{1} \gamma\right)\left(p^{0}+H_{1}+H_{1} \gamma\right) \\
& +\left(p^{0}+T a\right)\left(p^{0^{2}}+H_{1} \gamma\left(2 p^{0}+H_{1}(\alpha+\gamma)\right)\right) \delta,
\end{aligned}
$$

where $p^{0}=1+m$. The critical value of $R^{(\mathrm{Osc})}$ is found by minimizing with respect to $m$ and checking with Eq. (33) whether $\omega^{2}>0$ or not. Since Eq. (33) is quadratic in $\omega^{2}$, there may exist two positive values of $\omega^{2}$, then the critical values of $R^{(\mathrm{Osc})}$ between the two positive values of $\omega^{2}$ are compared, and the least value between them would be considered. Amplitudes at the order $\phi$ are found to be related in the form

$$
\begin{aligned}
& C_{1}=\frac{-\gamma H_{1} \sqrt{m}\left[\delta \omega_{0}\left\{p^{0}(1+\alpha)+H_{1}(\alpha+\gamma)\right\}+i\left\{p^{0}\left(p^{0}+H_{1}+H_{1} \gamma\right)-\alpha \delta^{2} \omega_{0}^{2}\right\}\right]}{\pi s^{0}} A_{1}, \\
& C_{1}^{*}=\frac{-\gamma H_{1} \sqrt{m}\left[\delta \omega_{0}\left\{p^{0}(1+\alpha)+H_{1}(\alpha+\gamma)\right\}-i\left\{p^{0}\left(p^{0}+H_{1}+H_{1} \gamma\right)-\alpha \delta^{2} \omega_{0}^{2}\right\}\right]}{\pi s^{0}} A_{1}^{*}, \\
& B_{1}=\frac{-\sqrt{m}\left[\delta \omega_{0}\left\{p^{0^{2}}+H_{1} \gamma\left(2 p^{0}+H_{1}(\alpha+\gamma)\right)+\alpha^{2} \delta^{2} \omega_{0}^{2}\right\}+i\left\{p^{0}\left(p^{0}+H_{1} \gamma\right)\left(p^{0}+H_{1}+H_{1} \gamma\right)+\left(p^{0}+H_{1}\right) \alpha^{2} \delta^{2} \omega_{0}^{2}\right\}\right]}{\pi s^{0}} A_{1}, \\
& B_{1}^{*}=\frac{-\sqrt{m}\left[\delta \omega_{0}\left\{p^{0^{2}}+H_{1} \gamma\left(2 p^{0}+H_{1}(\alpha+\gamma)\right)+\alpha^{2} \delta^{2} \omega_{0}^{2}\right\}-i\left\{p^{0}\left(p^{0}+H_{1} \gamma\right)\left(p^{0}+H_{1}+H_{1} \gamma\right)+\left(p^{0}+H_{1}\right) \alpha^{2} \delta^{2} \omega_{0}^{2}\right\}\right]}{\pi s^{0}} A_{1}^{*},
\end{aligned}
$$

where

$$
s^{0}=p^{0^{2}}\left(p^{0}+H_{1}+H_{1} \gamma\right)^{2}+\delta^{2} \omega_{0}^{2}\left\{\alpha^{2} \delta^{2} \omega_{0}^{2}+p^{0^{2}}\left(1+\alpha^{2}\right)+H_{1}^{2}(\alpha+\gamma)^{2}+2 H_{1} p^{0}\left(\alpha^{2}+\gamma\right)\right\} .
$$

At the second order in $\phi$, the system of linear partial differential equations is obtained as

$$
L_{1}^{(\mathrm{Osc})} \vec{\zeta}_{2}=N_{21}, \quad L_{2}^{(\mathrm{Osc})} \vec{\zeta}_{2}=N_{22}, \quad L_{3}^{(\mathrm{Osc})} \vec{\zeta}_{2}=N_{23},
$$

where

$$
\begin{aligned}
& N_{21}=-2 \frac{\partial}{\partial \tau_{0}}\left(\omega_{0} \frac{\partial}{\partial \tilde{t}}+1\right) \nabla^{2} \psi^{(1)}-R_{\mathrm{TD}_{\mathrm{cr}}} \frac{\partial^{2} T_{\mathrm{f}}^{(1)}}{\partial \tau_{0} \partial x}, \\
& N_{22}=\frac{\partial \psi^{(1)}}{\partial x} \frac{\partial T_{\mathrm{f}}^{(1)}}{\partial z}-\frac{\partial \psi^{(1)}}{\partial z} \frac{\partial T_{\mathrm{f}}^{(1)}}{\partial x}-\chi \frac{\partial T_{\mathrm{f}}^{(1)}}{\partial \tau_{0}}, \\
& N_{23}=-\alpha \chi \frac{\partial T_{\mathrm{s}}^{(1)}}{\partial \tau_{0}} .
\end{aligned}
$$

The complete solution at the second order in $\phi$ contains two parts, namely the complementary function corresponding to homogeneous part and the particular integral corresponding to inhomogeneous terms appearing in the right-hand side of Eq. (35). The complementary function resembles the first-order solution that balances the linear theory. The particular solutions are determined by removing the unwanted terms pertaining to resonance appearing 
in the form $\tilde{t} \sin (\tilde{t}) \sin (a x) \sin (\pi z)$ or $\tilde{t} \cos (\tilde{t}) \sin (a x) \sin (\pi z)$. Resonance is prevented by ignoring the slow timescale $\tau_{0}$, i.e. we set $\partial A_{1} / \partial \tau_{0}=0$. Hence, the complete solution at the second order in $\phi$ is represented as

$$
\begin{aligned}
& \psi^{(2)}=2 i\left(A_{2} e^{i \tilde{t}}-A_{2}^{*} e^{-i \tilde{t}}\right) \sin a x \sin \pi z, \\
& T_{\mathrm{f}}^{(2)}=2\left(B_{2} e^{i \tilde{t}}+B_{2}^{*} e^{-i \tilde{t}}\right) \cos a x \sin \pi z+\left(M_{2}+M_{1} e^{2 i \tilde{t}}+M_{1}^{*} e^{-2 i \tilde{t}}\right) \sin 2 \pi z, \\
& T_{\mathrm{s}}^{(2)}=2\left(C_{1} e^{i \tilde{t}}+C_{1}^{*} e^{-i \tilde{t}}\right) \cos a x \sin \pi z+\left(F_{2}+F_{1} e^{2 i \tilde{t}}+F_{1}^{*} e^{-2 i \tilde{t}}\right) \sin 2 \pi z,
\end{aligned}
$$

where

$$
\begin{aligned}
& F_{1}=\frac{-m H_{1} \gamma\left(r_{3}+i r_{4}\right)}{\pi s^{0}\left(p_{1}^{2}+p_{2}^{2}\right)} A_{1}^{2}, \quad F_{1}^{*}=\frac{-m H_{1} \gamma\left(r_{3}-i r_{4}\right)}{\pi s^{0}\left(p_{1}^{2}+p_{2}^{2}\right)}\left(A_{1}^{*}\right)^{2}, \\
& F_{2}=\frac{-m H_{1} \gamma\left\{p^{0}\left(p^{0}+H_{1} \gamma\right)\left(p^{0}+H_{1}+H_{1} \gamma\right)+\left(p^{0}+H_{1}\right) \alpha^{2} \delta^{2} \omega_{0}^{2}\right\}}{\pi s^{0}\left(4+H_{1} \gamma+H_{1}\right)} A_{1} A_{1}^{*}, \\
& M_{1}=\frac{m\left(r_{1}+i r_{2}\right)}{\pi s^{0}\left(p_{1}^{2}+p_{2}^{2}\right)} A_{1}^{2}, \quad M_{1}^{*}=\frac{m\left(r_{1}-i r_{2}\right)}{\pi s^{0}\left(p_{1}^{2}+p_{2}^{2}\right)}\left(A_{1}^{*}\right)^{2}, \\
& M_{2}=\frac{-m\left(4+H_{1} \gamma\right)\left\{p^{0}\left(p^{0}+H_{1} \gamma\right)\left(p^{0}+H_{1}+H_{1} \gamma\right)+\left(p^{0}+H_{1}\right) \alpha^{2} \delta^{2} \omega_{0}^{2}\right\}}{\pi s^{0}\left(4+H_{1} \gamma+H_{1}\right)} A_{1} A_{1}^{*},
\end{aligned}
$$

with

$$
\begin{aligned}
p_{1}=2 & {\left[4+H_{1}(1+\gamma)-\alpha \delta^{2} \omega_{0}^{2}\right], \quad p_{2}=\delta \omega_{0}\left[4(1+\alpha)+H_{1}(\alpha+\gamma)\right], } \\
r_{1}= & \left(\delta \omega_{0} \alpha p_{1}-\left(4+H_{1} \gamma\right) p_{2}\right) \delta \omega_{0}\left\{p^{0^{2}}+H_{1} \gamma\left(2 p^{0}+H_{1}(\alpha+\gamma)\right)+\alpha^{2} \delta^{2} \omega_{0}^{2}\right\} \\
& +\left(2 \alpha \delta \omega_{0} p_{2}+p_{1}\left(4+H_{1} \gamma\right)\right)\left\{p^{0}\left(p^{0}+H_{1} \gamma\right)\left(p^{0}+H_{1}+H_{1} \gamma\right)+\left(p^{0}+H_{1}\right) \alpha^{2} \delta^{2} \omega_{0}^{2}\right\}, \\
r_{2}= & \left.\delta \omega_{0} \alpha p_{1}-\left(4+H_{1} \gamma\right) p_{2}\right\}\left\{p^{0}\left(p^{0}+H_{1} \gamma\right)\left(p^{0}+H_{1}+H_{1} \gamma\right)+\left(p^{0}+H_{1}\right) \alpha^{2} \delta^{2} \omega_{0}^{2}\right\} \\
& -\delta \omega_{0}\left\{2 \alpha \delta \omega_{0} p_{2}+p_{1}\left(4+H_{1} \gamma\right)\right\}\left\{p^{0^{2}}+H_{1} \gamma\left(2 p^{0}+H_{1}(\alpha+\gamma)\right)+\alpha^{2} \delta^{2} \omega_{0}^{2}\right\}, \\
r_{3}= & p_{2} \delta \omega_{0}\left\{p^{0^{2}}+H_{1} \gamma\left(2 p^{0}+H_{1}(\alpha+\gamma)\right)+\alpha^{2} \delta^{2} \omega_{0}^{2}\right\} \\
& -p_{1}\left\{p^{0}\left(p^{0}+H_{1} \gamma\right)\left(p^{0}+H_{1}+H_{1} \gamma\right)+\left(p^{0}+H_{1}\right) \alpha^{2} \delta^{2} \omega_{0}^{2}\right\}, \\
r_{4}= & p_{2}\left\{p^{0}\left(p^{0}+H_{1} \gamma\right)\left(p^{0}+H_{1}+H_{1} \gamma\right)+\left(p^{0}+H_{1}\right) \alpha^{2} \delta^{2} \omega_{0}^{2}\right\} \\
& +p_{1} \delta \omega_{0}\left\{p^{0^{2}}+H_{1} \gamma\left(2 p^{0}+H_{1}(\alpha+\gamma)\right)+\alpha^{2} \delta^{2} \omega_{0}^{2}\right\} .
\end{aligned}
$$

The relation among the amplitudes is similar to Eq. (34).

At the third order in $\phi$, the system of partial differential equations is provided in the form

$$
L_{1}^{(\mathrm{Osc})} \vec{\zeta}_{3}=N_{31}, \quad L_{2}^{(\mathrm{Osc})} \vec{\zeta}_{3}=N_{32}, \quad L_{3}^{(\mathrm{Osc})} \vec{\zeta}_{3}=N_{33},
$$

where

$$
\begin{aligned}
N_{31}= & -R_{\mathrm{TD}_{\mathrm{cr}}}\left\{\left(\omega_{0} \frac{\partial}{\partial \tilde{t}}+1\right) \frac{\partial T_{\mathrm{f}}^{(1)}}{\partial x}+\frac{\partial}{\partial \tau_{0}}\left(\frac{\partial T_{\mathrm{f}}^{(2)}}{\partial x}\right)+\frac{\partial}{\partial \tau}\left(\frac{\partial T_{\mathrm{f}}^{(1)}}{\partial x}\right)\right\} \\
& -\frac{\partial^{2}}{\partial \tau_{0}^{2}}\left(\nabla^{2} \psi^{(1)}\right)-2 \frac{\partial}{\partial \tau_{0}}\left(\omega_{0} \frac{\partial}{\partial \tilde{t}}+1\right) \nabla^{2} \psi^{(2)}-2 \frac{\partial}{\partial \tau}\left(\omega_{0} \frac{\partial}{\partial \tilde{t}}+1\right) \nabla^{2} \psi^{(1)},
\end{aligned}
$$




$$
\begin{aligned}
& N_{32}=-\chi\left(\frac{\partial T_{\mathrm{f}}^{(2)}}{\partial \tau_{0}}+\frac{\partial T_{\mathrm{f}}^{(1)}}{\partial \tau}\right)+\frac{\partial \psi^{(1)}}{\partial x} \frac{\partial T_{\mathrm{f}}^{(2)}}{\partial z}-\frac{\partial \psi^{(1)}}{\partial z} \frac{\partial T_{\mathrm{f}}^{(2)}}{\partial x}+\frac{\partial \psi^{(2)}}{\partial x} \frac{\partial T_{\mathrm{f}}^{(1)}}{\partial z}-\frac{\partial \psi^{(2)}}{\partial z} \frac{\partial T_{\mathrm{f}}^{(1)}}{\partial x}, \\
& N_{33}=-\alpha \chi\left(\frac{\partial T_{\mathrm{s}}^{(2)}}{\partial \tau_{0}}+\frac{\partial T_{\mathrm{s}}^{(1)}}{\partial \tau}\right) .
\end{aligned}
$$

The system of equations at the third order in $\phi$ is converted into a single equation as

$$
\begin{aligned}
& \left\{\left(\chi \omega_{0} \frac{\partial}{\partial \tilde{t}}-\nabla^{2}\right)\left(\alpha \chi \omega_{0} \frac{\partial}{\partial \tilde{t}}-\nabla^{2}+\gamma H\right)+H\left(\alpha \chi \omega_{0} \frac{\partial}{\partial \tilde{t}}-\nabla^{2}\right)\right\} \\
& \quad \times\left\{\left(\left(\omega_{0} \frac{\partial}{\partial \tilde{t}}+1\right)^{2} \nabla^{2}+T a \frac{\partial^{2}}{\partial z^{2}}\right)\right\} \psi^{(3)} \\
& \quad-R_{\mathrm{TD}_{\mathrm{cr}}}\left(\omega_{0} \frac{\partial}{\partial \tilde{t}}+1\right)\left(\alpha \chi \omega_{0} \frac{\partial}{\partial \tilde{t}}-\nabla^{2}+\gamma H\right) \frac{\partial^{2} \psi^{(3)}}{\partial x^{2}}=\mathrm{NHT},
\end{aligned}
$$

where NHT represents the inhomogeneous terms which include the known solutions obtained at the previous orders. The system of linear partial differential equations obtained at the order $\phi^{3}$ will be the inhomogeneous version of the linear system determined at the order $\phi$. To make Eq. (38) solvable, we equate the coefficients of the inhomogeneous terms associated with resonance to zero to get the solvability condition in the form of following ordinary differential equation

$$
\frac{\mathrm{d} A}{\mathrm{~d} t}=\left[h_{21} \xi_{\mathrm{Osc}}-h_{31} A A^{*}\right] A,
$$

where

$$
A=\phi A_{1}, A^{*}=\phi A_{1}^{*}, \xi_{\mathrm{Osc}}=\phi^{2}=\left[\frac{R}{R_{\mathrm{cr}}^{(\mathrm{Osc})}}-1\right], h_{21}=h_{21}^{0}+i m_{21}, h_{31}=h_{31}^{0}+i m_{31} .
$$

The expressions for coefficients $h_{21}^{0}, m_{21}, h_{31}^{0}$ and $m_{31}$ are too lengthy and are not presented here. It is important to note that the coefficients $h_{21}^{0}, m_{21}, h_{31}^{0}$ and $m_{31}$ are functions of $H_{1}, \alpha, \gamma, T a, R_{\mathrm{cr}}^{(\mathrm{Osc})}, \delta, \omega_{0}$ and the scaled horizontal wavenumber $m$. Equation (39) represents the Ginzburg-Landau equation for the oscillatory case in which the original timescale $t=t^{\prime} / \chi$ has been employed. To study the amplitude fluctuations and frequency results, the complex amplitude $A$ and its conjugate $A^{*}$ are written in the exponential form as

$$
A=r e^{i \theta} \text {, and } A^{*}=r e^{-i \theta} \text {, with } r=|A| \text {, and } A A^{*}=r^{2} \text {. }
$$

Substituting Eq. (40) into (39) yields a coupling of two differential equations that corresponds to absolute value of the amplitude as well as frequency (phase) as follows:

$$
\begin{gathered}
h_{12}^{0} \frac{\mathrm{d} r}{\mathrm{~d} t}=\left[\xi_{\mathrm{Osc}}-h_{32}^{0} r^{2}\right] r, \\
\frac{\mathrm{d} \theta}{\mathrm{d} t}=m_{21} \xi_{\mathrm{Osc}}-m_{31} r^{2},
\end{gathered}
$$

where $h_{12}^{0}=1 / h_{21}^{0}$ and $h_{32}^{0}=h_{31}^{0} / h_{21}^{0}$. The stability of bifurcation is analysed based on the coefficient of the nonlinear term $h_{32}^{0}$. The positive values of $h_{32}^{0}$ correspond to forward (supercritical) bifurcation, and the negative values of $h_{32}^{0}$ correspond to inverse (subcritical) bifurcation. The point at which $h_{32}^{0}$ changes sign is recognized as the transition point from 
supercritical to subcritical motion. For the extremely critical values of $R$, at the steady state (post-transient state) Eq. (41) yields the solution for $r$ as follows:

$$
r=\left\{\begin{array}{lr}
0 & \forall R<R_{\mathrm{cr}}^{(\mathrm{Osc})} \\
\pm\left[\left(1 / h_{32}^{0}\right) \xi_{\mathrm{Osc}}\right]^{1 / 2} & \forall R \geq R_{\mathrm{cr}}^{(\mathrm{Osc})}
\end{array} .\right.
$$

By using Eq. (40), the steady-state (post-transient state) amplitude solution is provided as

$$
A= \pm\left[\left(1 / h_{32}^{0}\right) \xi_{\mathrm{Osc}}\right]^{1 / 2} e^{i \dot{\theta} t} .
$$

Equations (31) and (44) (i.e. post-transient state amplitude solution and the first-order solution) reveal the existence of Hopf bifurcation in the case of oscillatory convection. Hopf bifurcation is a local bifurcation in which a fixed point of a dynamical system loses stability, as a pair of complex conjugate eigenvalues of the linearization around the fixed point crosses the complex plane imaginary axis.

Substituting Eq. (43) into Eq. (42) gives an expression for the frequency in the form

$$
\dot{\theta}=\frac{\mathrm{d} \theta}{\mathrm{d} t}=\xi_{\mathrm{Osc}}\left[m_{21}-m_{31}\left(1 / h_{32}^{0}\right)\right] \text {. }
$$

\section{Heat Transfer}

The heat transfer is studied in terms of Nusselt number. The average Nusselt number for nonlinear oscillatory convection is defined as

$$
{\overline{N u_{f}}}^{(\mathrm{Osc})}=\frac{1}{\left(2 \pi / a_{\mathrm{cr}}\right)\left(2 \pi / \omega_{0}\right)}\left[\int_{0}^{\left(2 \pi / \omega_{0}\right)} \int_{0}^{\left(2 \pi / a_{\mathrm{cr}}\right)}\left(-\partial T_{f} / \partial z\right) \mathrm{d} x \mathrm{~d} t\right]_{z=0} .
$$

Substituting the post-transient state amplitude solutions corresponding to nonlinear oscillatory convection in the absence of slow space scale yields the average Nusselt number for the oscillatory convection in the form

$$
\begin{aligned}
& \overline{N u}_{\mathrm{f}}{ }^{(\mathrm{Osc})}=1 \\
& +\frac{2 m\left(4+H_{1} \gamma\right)\left(1 / h_{32}^{0}\right)\left\{p^{0}\left(p^{0}+H_{1} \gamma\right)\left(p^{0}+H_{1}+H_{1} \gamma\right)+\left(p^{0}+H_{1}\right) \alpha^{2} \delta^{2} \omega_{0}^{2}\right\} \xi_{\mathrm{Osc}}}{\left(4+H_{1}(1+\gamma)\right) s^{0}} . \\
& \quad \forall R \geq R_{\mathrm{cr}}^{(\mathrm{Osc})}
\end{aligned}
$$

When $H_{1}=0$ (LTE model), Eq. (47) yields

$$
{\overline{N u_{\mathrm{f}}}}^{(\mathrm{Osc})}=1+\frac{2 m p^{0}\left(1 / h_{32}^{0}\right)}{\left(p^{0^{2}}+\delta^{2} \omega_{0}^{2}\right)}\left[\frac{R}{R_{\mathrm{cr}}^{(\mathrm{Osc})}}-1\right], \quad \forall R \geq R_{\mathrm{cr}}^{(\mathrm{Osc})} .
$$

and coincides with Vadasz (1998).

\section{Results and Discussion}

The effect of local thermal non-equilibrium (LTNE) on weak nonlinear stability of both stationary and oscillatory porous convection in a rotating fluid-saturated porous layer is investigated. By employing the multi-scale method, the Ginzburg-Landau amplitude equations are derived specifying the pitchfork and Hopf bifurcations to the cases of stationary and 
oscillatory finite amplitude convection, respectively, at their corresponding critical values of the Darcy-Rayleigh number.

Since the weak nonlinear stability analysis is based on the linear instability analysis, some additional results are given. Figure $1 \mathrm{a}-\mathrm{c}$ shows the stability maps in the $(\delta, T a)$-plane demarcating the regions of stationary and oscillatory convection for LTE and LTNE cases corresponding to some specified values of the parameters. By introducing local thermal non-equilibrium, we can decrease the co-dimension-2 points and the region of oscillatory convection with the increase in the region of stationary convection in the $(\delta, T a)$-plane. The region below the continuous curve corresponds to oscillatory convection and the above it represents the stationary convection. Also, limit of possible oscillatory convection is identified by dotted line, while the continuous curve shows the limit where stationary and oscillatory critical Rayleigh numbers are the same identifying to co-dimension-2 point (i.e. point of interaction of stationary and oscillatory modes). Below the dotted curve and above the continuous curve, oscillatory convection can occur but it does not happen since $R_{\mathrm{cr}}^{(\mathrm{St})}<R_{\mathrm{cr}}^{(\mathrm{Osc})}$. As LTNE parameters $H(=0,100), \alpha(=0,1)$ and $\gamma(=0,5)$ increase, the region of stationary convection enhances, while that of oscillatory convection decreases and also the co-dimension- 2 points as well.

The Pitchfork bifurcation occurs at stationary critical Darcy-Rayleigh number in the case of nonlinear stationary convection. Figure 2 presents the variation of stationary amplitude at Pitchfork bifurcation with scaled Darcy-Rayleigh number for LTE and LTNE cases for different values of Darcy-Taylor number $T a(=70,100,130)$. For a fixed value of $T a$, there exists a transition value of the scaled Darcy-Rayleigh number, denoted by $R^{\diamond}$, at which the amplitudes of Pitchfork bifurcation for both LTE and LTNE cases will be equal when $R>R_{\mathrm{cr}}^{(\mathrm{St})}$. Besides, the amplitude of Pitchfork bifurcation at $R>R_{\mathrm{cr}}^{(\mathrm{St})}$ for the LTE case is larger than that of LTNE case when $R<R^{\diamond}$, while the trend gets reversed for $R>R^{\diamond}$.

Heat transfer corresponding to nonlinear stationary convection is evaluated in terms of thermal Nusselt number and shown in Fig. 3a-d. These figures unveil the variation of average thermal Nusselt number as a function of scaled thermal Darcy-Rayleigh number $R$ for different values of parameters. The average thermal Nusselt number decreases with $T a(=50,75,100)$ at a fixed value of the scaled Darcy-Rayleigh number, indicating the retarding effect of rotation on heat transfer. An increase in the value of $\gamma(=0,5,10)$ is to increase the heat transfer while increasing $H(=0,50,100)$ produces an opposite effect on heat transfer. In conclusion, LTNE produces retarding effect as well as accelerating effect on heat transfer in the case of stationary convection in opposition to the local thermal equilibrium wherein only retarding effects are observed on heat flux for stationary convection as in LTE case stationary Rayleigh number depends only on Darcy-Taylor number Ta and it produces retarding effect on heat flux but in LTNE case stationary Rayleigh number depends on Darcy-Taylor number $T a$ and LTNE parameters $H, \gamma$ where $T a$ and $H$ decrease heat flux while $\gamma$ enhances the heat flux. Therefore, with LTNE effects, heat transfer corresponding to stationary convection is decreased compared to LTE case.

The positive and negative values of nonlinear amplitude coefficient $h_{32}^{0}$ appearing in the Ginzburg-Landau equation refer to forward and inverse bifurcations, respectively. There exists a point at which the sign of $h_{32}^{0}$ changes, called transition point from supercritical to subcritical convection. Figure 4 shows the variation of $h_{32}^{0}$ as a function of $\delta$ for LTE $(T a=40, H=0, \alpha=0=\gamma)$ and $\operatorname{LTNE}(T a=40, H=30, \alpha=0.5, \gamma=0.8)$ cases. It is seen that there exists a maximum value of $\delta, \delta \max =6.66305$ for the LTE and $\delta_{\max }=7.8862$ for the LTNE models, reliable with the condition $\omega_{0}^{2} \geq 0$ for the considered 

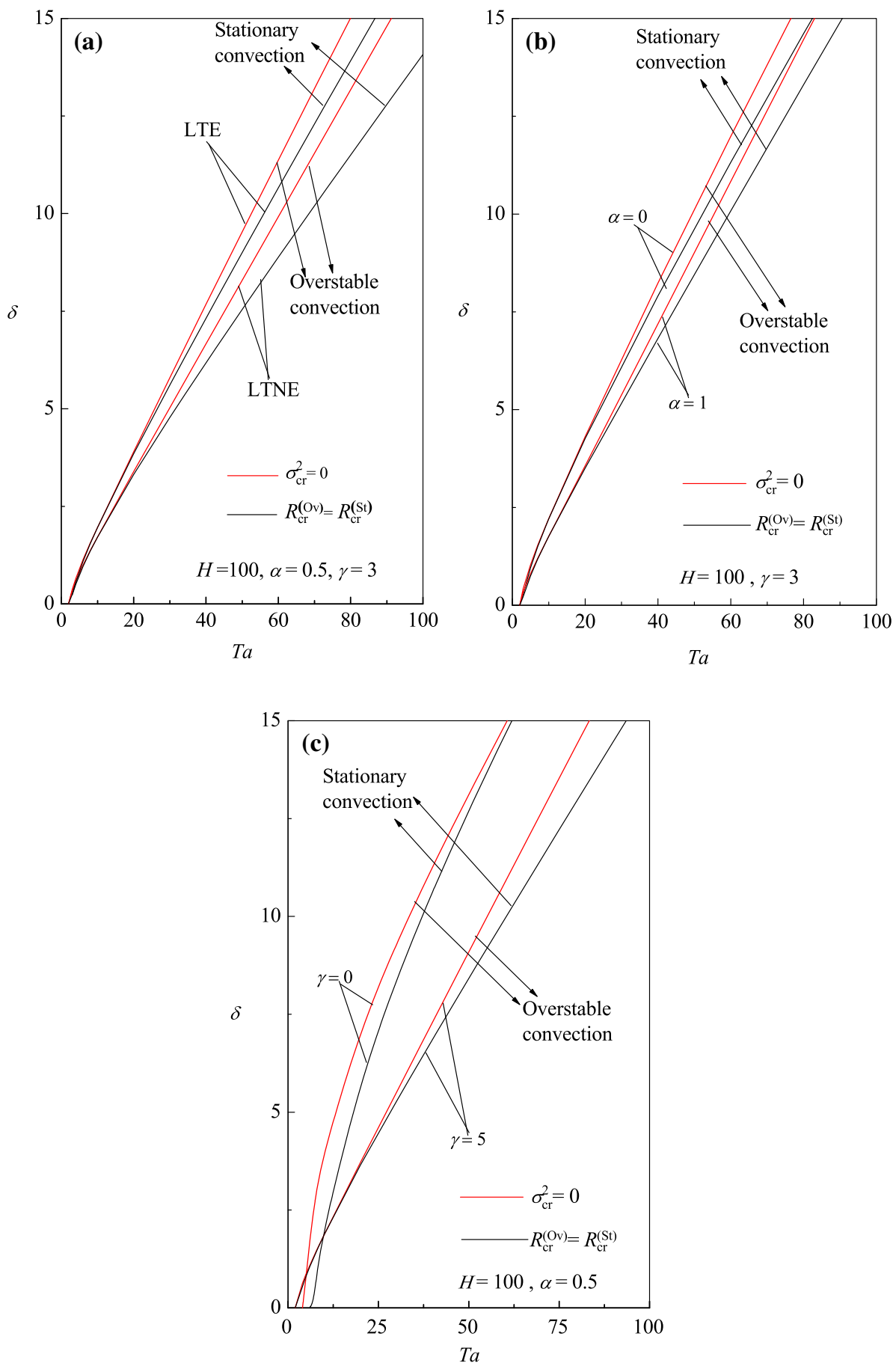

Fig. 1 The stability maps on the $(\delta, T a)$ - plane showing the division of the plane into zones of stationary and oscillatory convection 
Fig. 2 Stationary amplitude at Pitchfork bifurcation versus scaled Darcy-Rayleigh number for different values of Darcy Taylor number

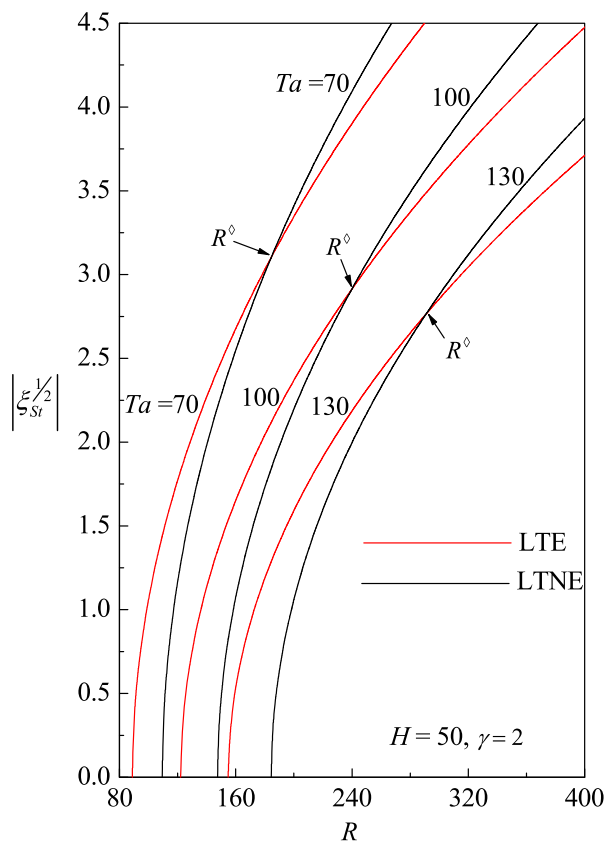

set of values. Hence, it is evident that forward bifurcation exists for $\delta<\delta_{t}$, where $\delta_{t}$ is the value of $\delta$ corresponding to transition point from supercritical to subcritical motion and inverse bifurcation for $\delta_{t}<\delta<\delta_{\max }$. Moreover, there exist a point at which $h_{32}^{0}$ diverges called the co-dimension- 2 point and at least one point in the region $\delta>\delta \max$ at which the sign of $h_{32}^{0}$ changes that corresponds to the non-oscillatory region (i.e. $\omega_{0}^{2}<0$ ).

When $h_{32}^{0}=0$, it marks a transition between supercritical and subcritical convection. For this purpose, following Rees (2009), the linear and nonlinear oscillatory neutral curves are illustrated in Fig. 5a-f reliable with the condition $\omega_{0}^{2} \geq 0$. For each wave number, the dashed curve corresponds to the least value of the Darcy-Rayleigh number for which weak nonlinear convection occurs and the linear stability curve (continuous curve) lies above it. For wave numbers higher than that given by the point labelled (B), the nonlinear solution bifurcates supercritically while for lower values of wave numbers the bifurcation is subcritical. The point at which the nonlinear neutral curve branches off the linear neutral curve is termed as quartic point; a terminology used by Rees (2009). The quartic point increases with increased values of $T a=40$ and 70 (Fig. 5a, b), $H=0$ and 50 (Fig. 5c, d) and $\alpha=0$ and 1 (Fig. 5e), while it decreases slightly with increasing $\gamma=0$ and 5 (Fig. 5f). Values of $R^{(\mathrm{Osc})}$ and $m^{1 / 2}$ corresponding to the labelled points [i.e. (A), (B) and (C)] in Fig. 5a-f are listed in Table 1. The labelled points (A), (B) and (C) in Fig. 5a-f represent the minimum of linear curve, quartic point and the minimum of nonlinear curve, respectively. When the initial disturbance is sufficiently large, nonlinear oscillatory solutions exist for the minimum value of Rayleigh number less than that of the minimum value of linear oscillatory solution.

Figure $6 \mathrm{a}-\mathrm{c}$ displays the variation of $\delta_{t}$ and $\delta_{\max }$ with $T a$ for LTE case as well as LTNE case. From Fig. 6a-c, it is unveiled that there exists a transition value of Darcy-Taylor number, denoted by $T a^{\diamond}$ (corresponding value of $\delta$ is denoted by $\delta^{\diamond}$ ), at which $\delta_{t}=\delta_{\max }=\delta^{\diamond}$. We can observe that $\delta_{t}<\delta_{\max }$ for $T a>T a^{\diamond}$ and $\delta_{t}>\delta_{\max }$ for $T a<T a^{\diamond}$. The significance of the existence of transition value of the Darcy-Taylor number $T a^{\diamond}$ is to produce forward 

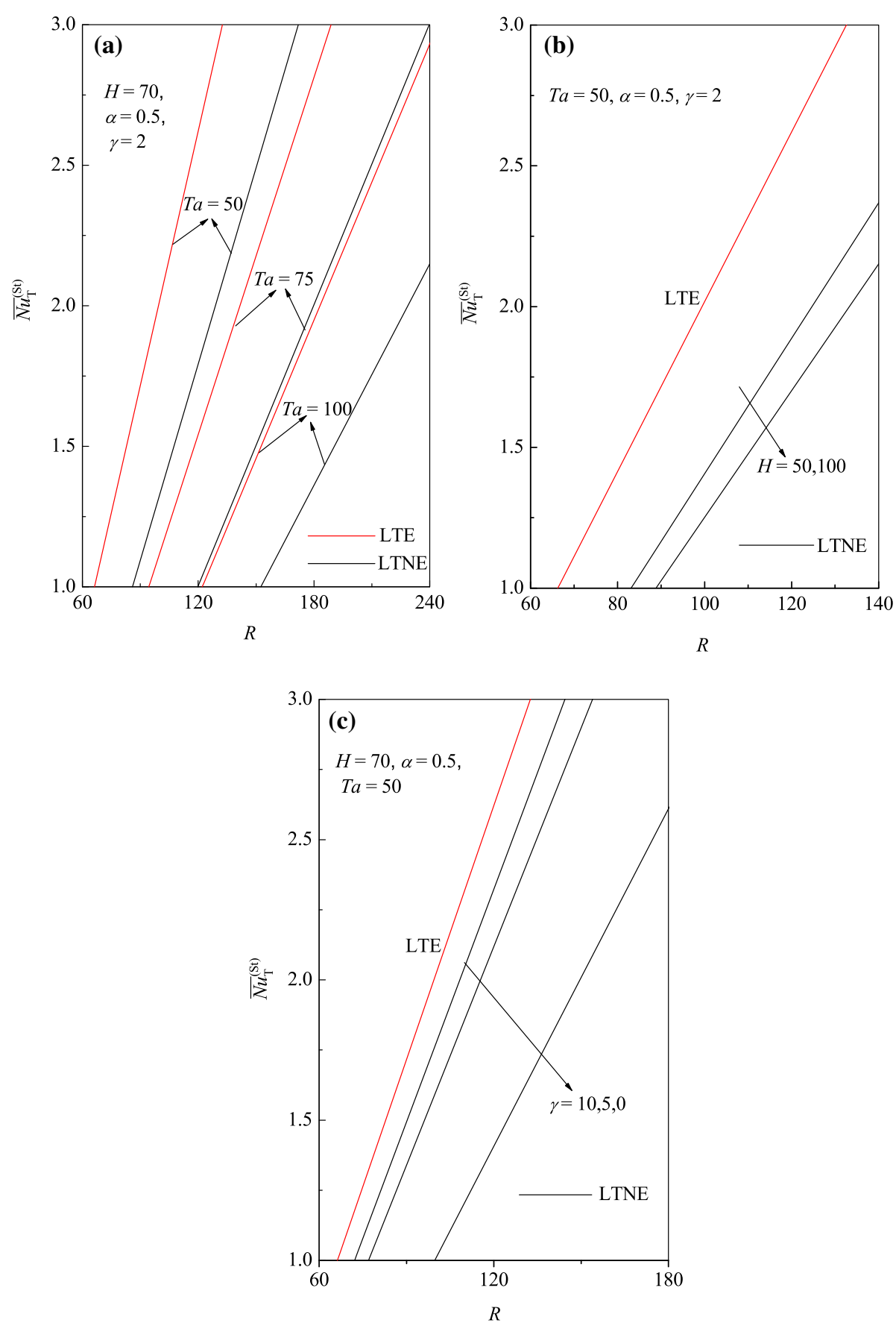

Fig. 3 Variation of steady case Nusselt number with scaled Darcy-Rayleigh number R 
Fig. 4 Variation of nonlinear term coefficient $h_{32}^{0}$ as a function of $\delta$

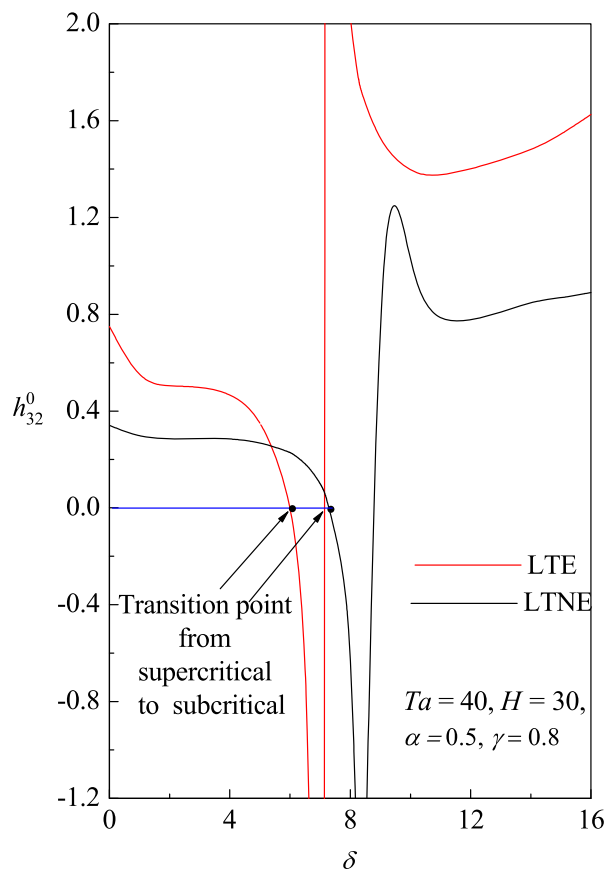

bifurcation with $T a<T a^{\diamond}$ for the whole domain of oscillatory convection. As $H$ increases, $\delta_{t}$ and $\delta_{\max }$ increase for any fixed value of $T a$. In addition, the transition value $T a^{\diamond}$ also increases with increasing $H$; thereby, the region of forward bifurcation for $T a<T a^{\diamond}$ will be enhanced compared to the region of forward bifurcation for $T a<T a^{\diamond}$ observed in the case of local thermal equilibrium. The pattern will be opposite with the variation of the parameters $\alpha$ and $\gamma$.

Figure $7 \mathrm{a}-\mathrm{d}$ represents the variation of post-transient amplitude in terms of $\log _{10}(|A| / \phi)$ as a function of $\delta$ for different values of $\operatorname{Ta}(=40,70,100), H(=0,50,100), \gamma(=0,5,10)$ and $\alpha(=0,0.5,1)$. The curves in these figures show the asymptotical behaviour of amplitude as $\delta$ tending to $\delta_{t}$, and this will occur in the neighbourhood of co-dimension-2 point (CTP) and not exactly at that point. With increasing $\operatorname{Ta}(=40,70,100)$ and $H(=0,50,100)$, the transition point from supercritical to subcritical convection increases and the amplitude solution exhibits an asymptotical behaviour as $\delta$ close to the transition point $\delta_{t}$, and this fact is evident from Fig. 7a, b, respectively. The trend gets reversed in the case of an increase in the parameter $\gamma(=0,5,10)$, and this is observed in Fig. 7c. As shown in Fig. 7d, the variation of transition point from supercritical to subcritical convection with increasing $\alpha(=0,0.5,1)$ is insignificant and the finite amplitude solution diverges in the neighbourhood of the same transition point from supercritical to subcritical convection for different values of $\alpha$. For moderate values of $\delta$, the amplitude solution is inversely proportional to $\operatorname{Ta}(=40,70,100)$ at any fixed value of $\delta$. With an increase in the values of $H(=0,50,100)$ and $\gamma(=0,5,10)$, it is observed that the finite amplitude curves will cross over to each other at the moderate values of $\delta$ and thereafter the effect of $H$ and $\gamma$ on the finite amplitude solution gets reversed. Hence, from Fig. 7a-d, we can conclude that the LTNE model enhances the decreasing region of post-transient amplitude when compared to the region observed with the LTE model.

The post-transient nonlinear frequency correction is determined by using the results of post-transient finite amplitude solution. The variation of post-transient nonlinear frequency 

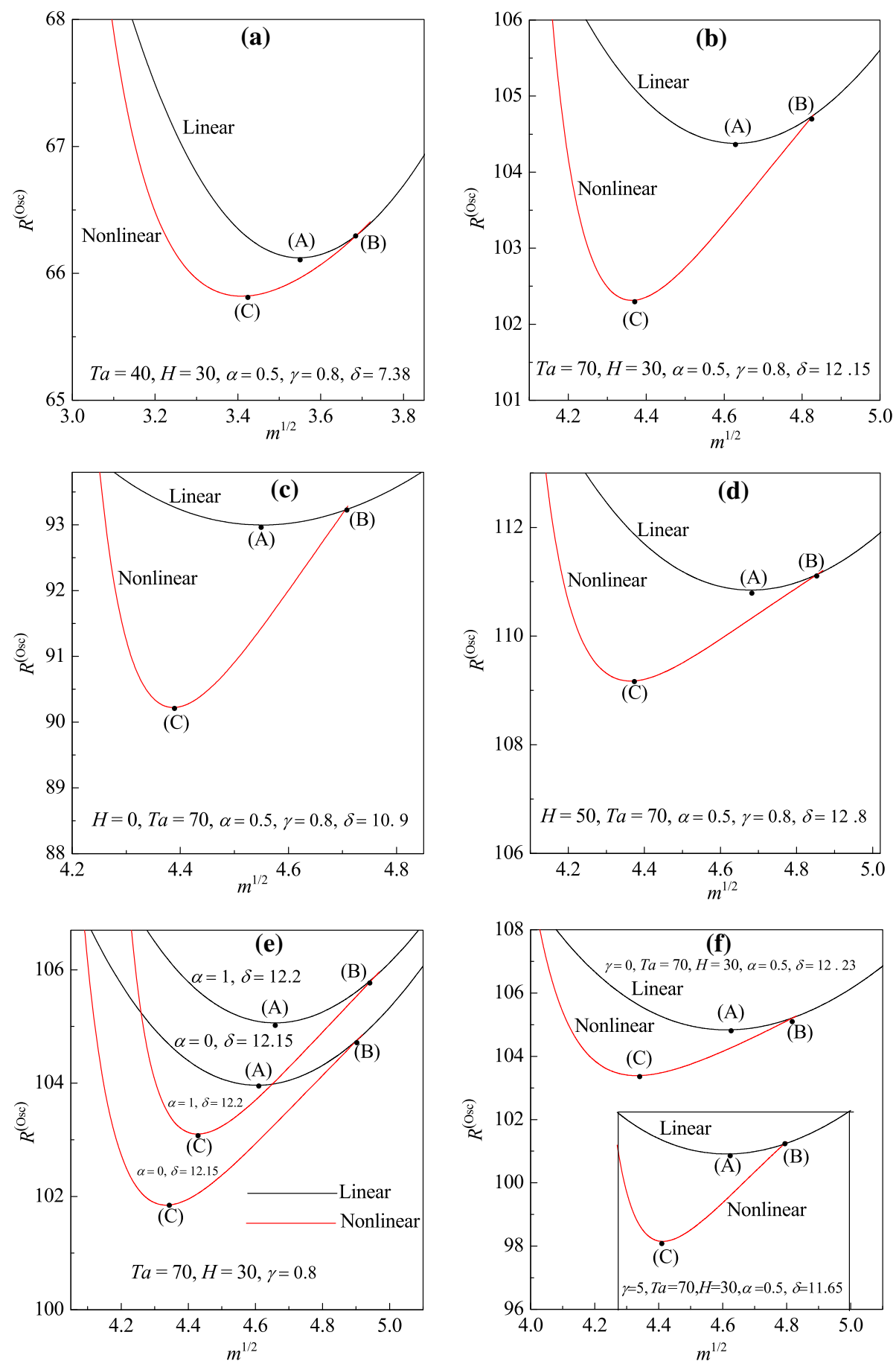

Fig. 5 Neutral curves for $R^{(\mathrm{Osc})}$ versus $m^{1 / 2}$. Bullets denote the specific points: $A$ the minimum of the linear curve; $B$ the quartic point (i.e. where the nonlinear curve branches off the linear curve); $C$ the minimum of the nonlinear curve 
Table 1 Values of $R^{(\mathrm{Osc})}$ and $m^{1 / 2}$ corresponding to the labelled points in Fig. 5a-f

\begin{tabular}{|c|c|c|c|}
\hline Figure & Point & $R^{(\mathrm{Osc})}$ & $m^{1 / 2}$ \\
\hline \multirow[t]{3}{*}{$5 \mathrm{a}$} & (A) & 66.1293 & 3.5452 \\
\hline & (B) & 66.2901 & 3.6814 \\
\hline & (C) & 65.8470 & 3.4228 \\
\hline \multirow[t]{3}{*}{$5 b$} & (A) & 104.3896 & 4.6286 \\
\hline & (B) & 104.7210 & 4.8217 \\
\hline & (C) & 102.3121 & 4.36889 \\
\hline \multirow[t]{3}{*}{$5 c$} & (A) & 92.9799 & 4.5531 \\
\hline & (B) & 93.2002 & 4.7031 \\
\hline & (C) & 90.2236 & 4.3873 \\
\hline \multirow[t]{3}{*}{$5 d$} & (A) & 110.8416 & 4.6814 \\
\hline & (B) & 111.1268 & 4.8524 \\
\hline & (C) & 109.1829 & 4.3733 \\
\hline \multirow[t]{6}{*}{$5 e$} & (A) & 103.9716 & 4.6217 \\
\hline & (B) & 104.6903 & 4.8934 \\
\hline & (C) & 101.8785 & 4.3455 \\
\hline & (A) & 105.0775 & 4.6591 \\
\hline & (B) & 105.7877 & 4.9420 \\
\hline & (C) & 103.1259 & 4.4312 \\
\hline \multirow[t]{6}{*}{$5 f$} & (A) & 104.8839 & 4.6220 \\
\hline & (B) & 105.2341 & 4.8117 \\
\hline & (C) & 103.4031 & 4.3423 \\
\hline & (A) & 100.9485 & 4.6168 \\
\hline & (B) & 101.2420 & 4.7926 \\
\hline & (C) & 98.1503 & 4.4238 \\
\hline
\end{tabular}

correction in terms of $\log _{10}\left(\dot{\theta} / \xi_{O s c}\right)$ is exhibited for different values of physical parameters as a function of $\delta$ (Fig. 8a-d). From these figures, one can identify that the nonlinear frequency correction is diverging as $\delta$ tending to $\delta_{t}$. Figure $8 \mathrm{a}, \mathrm{b}$ exhibits that an increase in $\mathrm{Ta}(=$ $40,70,100)$ and $H(=0,50,100)$ produces the retarding effect on post-transient nonlinear frequency correction at any fixed value of $\delta$. But Fig. 8c reveals the reverse trend on nonlinear frequency correction with the increase in $\gamma(=0,5,10)$. There is no significant effect of $\alpha(=0,0.5,1)$ on nonlinear frequency correction, and it is clear from Fig. $8 \mathrm{~d}$.

Heat transfer corresponding to nonlinear oscillatory convection is obtained via thermal Nusselt number in terms of $\log _{10}\left[\left(\overline{N u}^{(\mathrm{Ov})}-1\right) / \varepsilon^{2}\right]$ using finite amplitude solutions. Nusselt number for oscillatory convection diverges in the neighbourhood of the transition point from supercritical to subcritical convection. By implementing local thermal non-equilibrium, the region of enhancement on heat flux in the smaller $\delta$-domain with variation of rotation is increased compared to the region observed in the LTE case with the variation of rotation. Moreover, the LTNE parameter $\gamma$ also increases the region of enhancement on heat flux in the smaller $\delta$-domain. Figure $9 \mathrm{a}-\mathrm{d}$ discloses the variation of average thermal Nusselt number corresponding to nonlinear oscillatory convection in terms of $\log _{10}\left[\left(\overline{N u}^{(\mathrm{Ov})}-1\right) / \varepsilon^{2}\right]$ as a function of $\delta$ for various values of physical parameters. Figure 9a divulges that for minute 

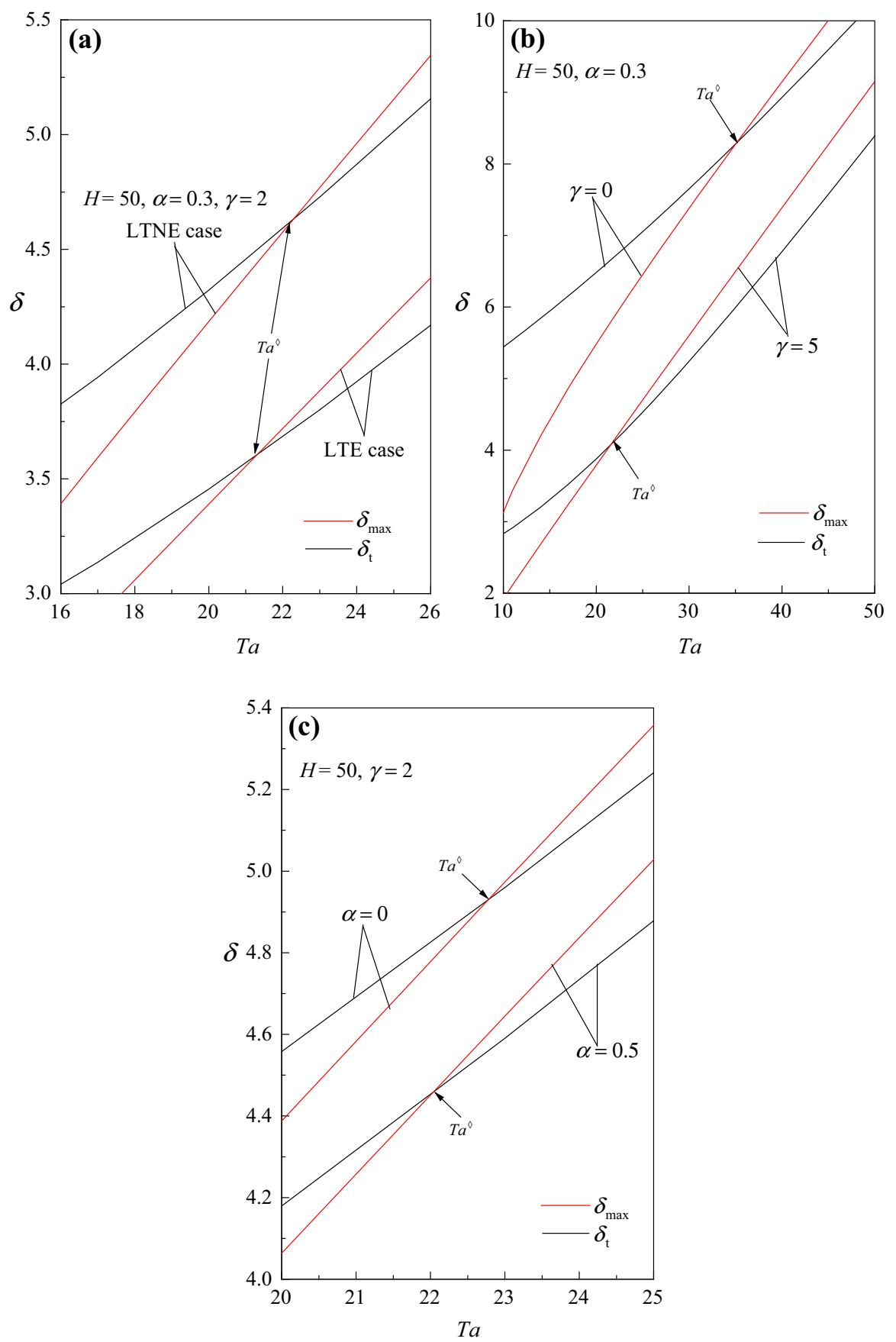

Fig. 6 Consequences of finite amplitude for oscillatory convection: $\delta_{t}$ and $\delta_{\max }$ as a function of $T a$ 

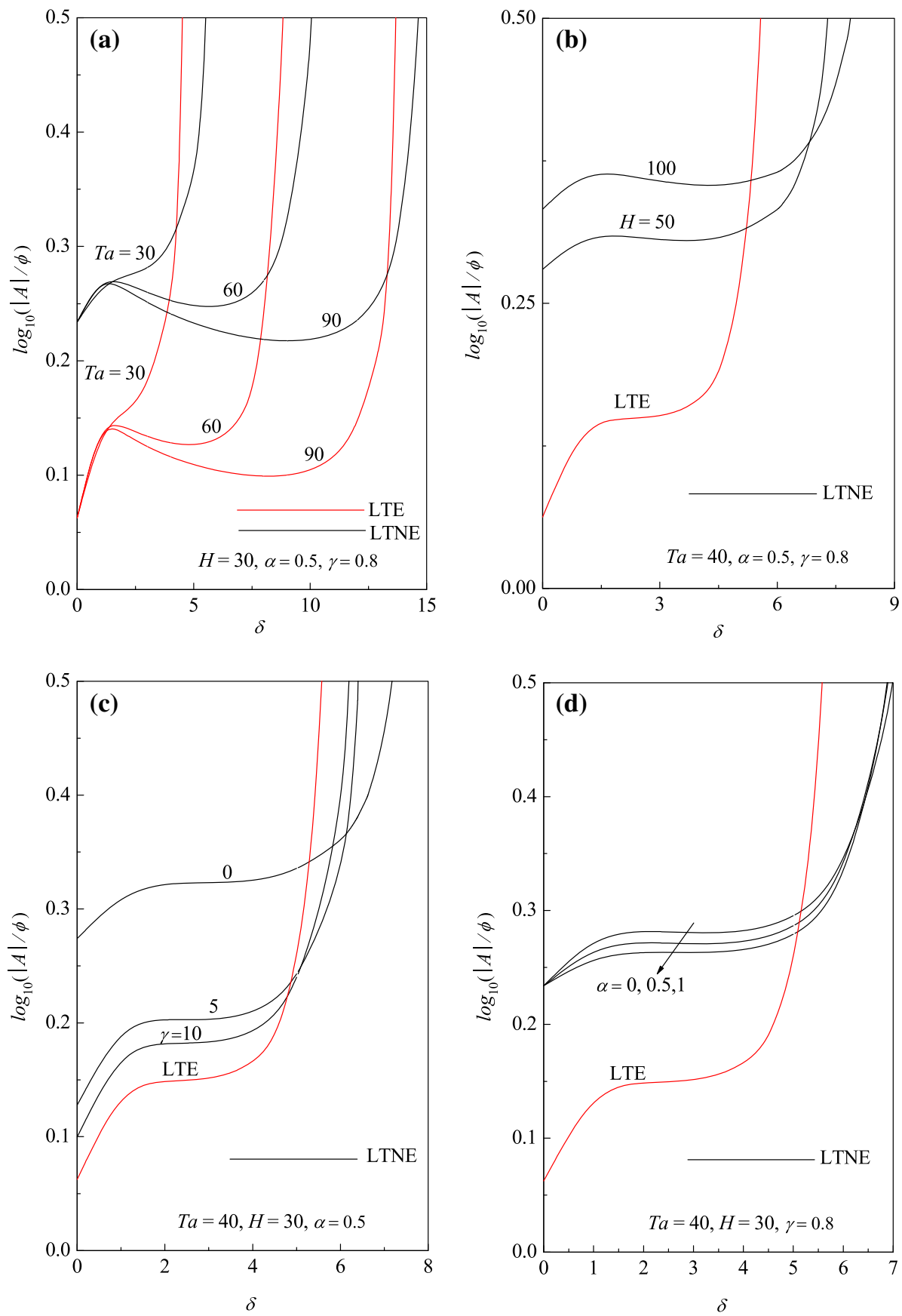

Fig. 7 Oscillatory post-transient amplitude as a function of scaled Vadasz number $\delta$ for different values of various physical parameters 

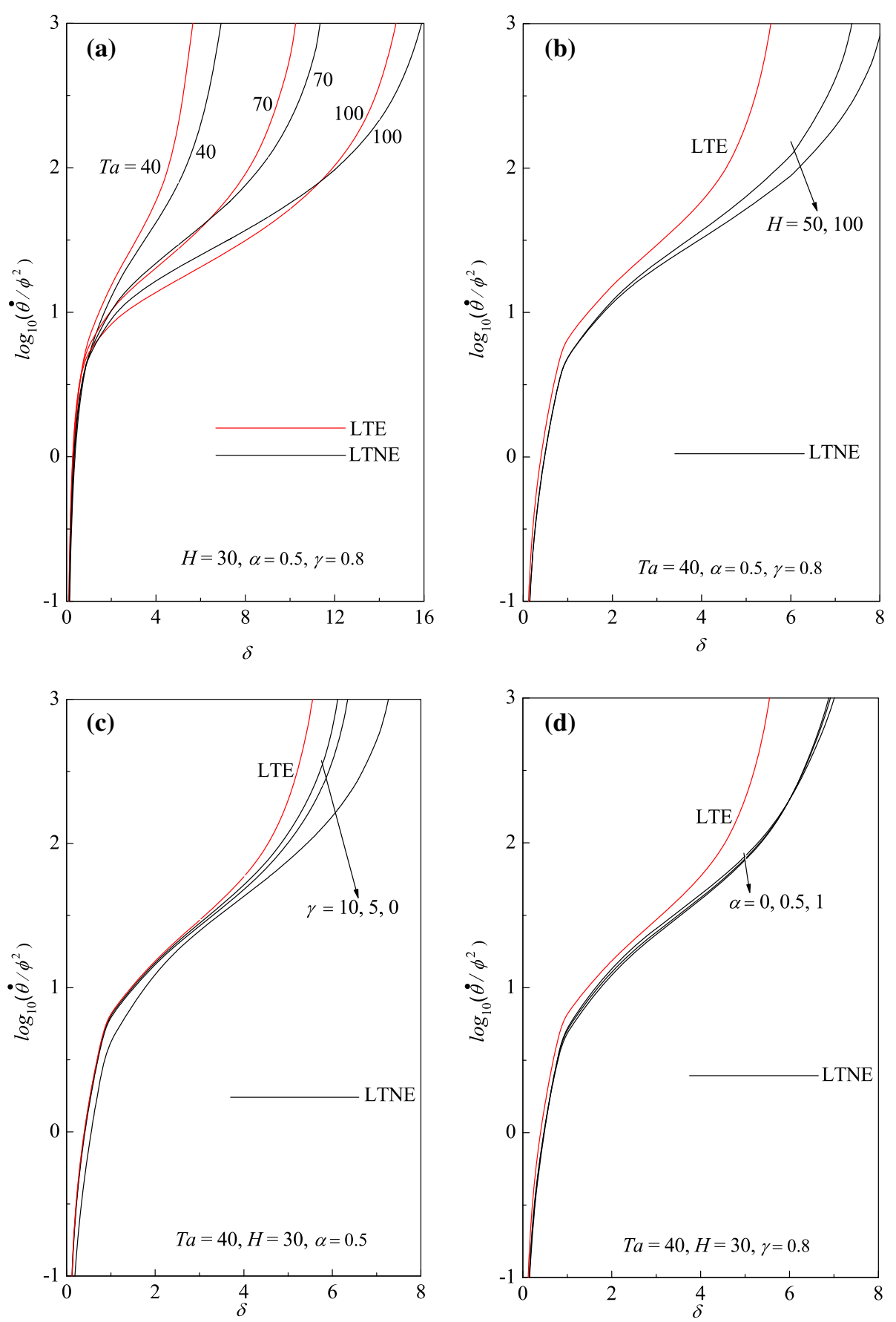

Fig. 8 Oscillatory post-transient nonlinear frequency correction as a function of scaled Vadasz number $\delta$ for different values of various physical parameters 

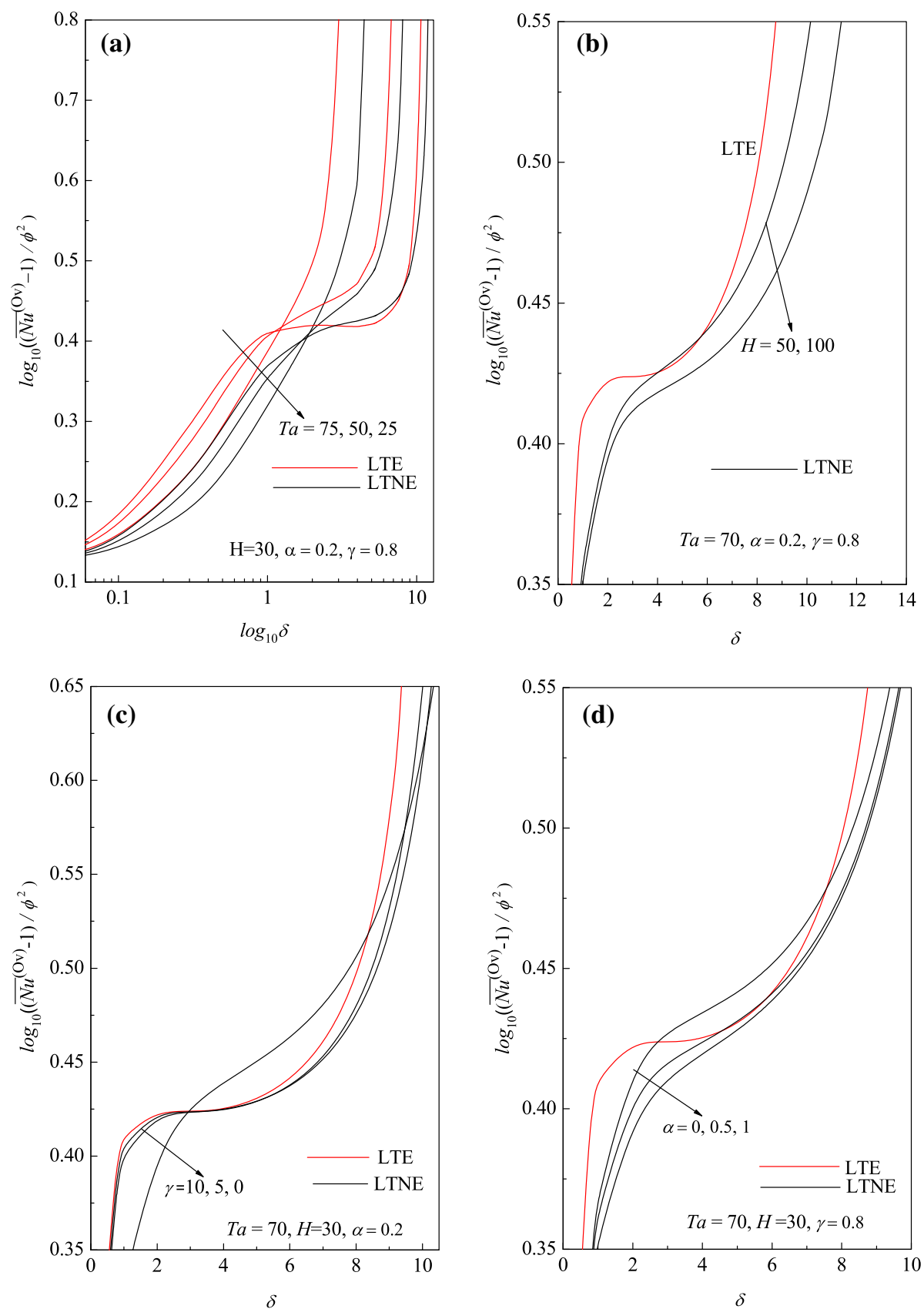

Fig. 9 Oscillatory post-transient Nusselt number versus scaled Vadasz number $\delta$ for various values of physical parameters 
values of $\delta$ but not too small, heat flux will be enhanced with the increase in $T a(=25,50,75)$ at the fixed value of $\delta$, signifying the accelerating upshot on heat transfer for oscillatory convection. But the trend gets reversed for most of the larger $\delta$-domain. The LTNE effects are mainly based on the interphase heat transfer coefficient $H$. The effect of parameter $H$ on heat flux for overstable convection is shown in Fig. 9b. As $H(=0,50,100)$ increases, the Nusselt number decreases at a particular value of $\delta$ over the entire $\delta$-domain, representing the retarding effect of $H$ on heat transfer for oscillatory convection. Figure 9c, d exposes the effect of $\gamma$ and $\alpha$, respectively, on heat flux corresponding to nonlinear oscillatory convection. With increasing $\gamma(=0,5,10)$, one can observe accelerating effect on heat transfer for oscillatory convection in the smaller $\delta$-domain and the scenario is opposite in the moderate $\delta$-domain. Even so, the pattern changes near the transition points from supercritical to subcritical motion, signifying the accelerating effect once again on heat transfer for oscillatory convection. As the parameter $\alpha(=0,0.5,1)$ increases, heat flux on oscillatory convection is decreased for the entire $\delta$-domain.

\section{Conclusions}

The impact of rotation and LTNE on both stationary and oscillatory weak nonlinear stability of an incompressible fluid-saturated porous layer is studied using the multi-scale method. By performing the linear instability analysis, the regions of stationary and oscillatory convection and co-dimension- 2 points are obtained by means of stability map. As the LTNE parameters $H, \alpha$ and $\gamma$ increase, the region of stationary convection enhances while the region of overstable convection decreases along with the co-dimension- 2 points. The bifurcation theory gives the complete picture of weak nonlinear stability analysis. The pitchfork and Hopf bifurcations are identified in stationary and in oscillatory convection, respectively, by the finite amplitude solutions. The quartic point at which the subcritical motion switches over to supercritical motion occurs is located for the governing parameters, and it increases with the increase in $T$ a, $H$ and $\alpha$ but decreases with increasing $\gamma$. The LTNE effect is to enforce the enhancement of region of forward bifurcation and the decreasing region of post-transient amplitude. Heat transfer corresponding to stationary convection is decreased in the presence of LTNE effects. The nonlinear frequency and heat flux have been evaluated by employing the finite amplitude solutions. The LTNE conditions yield intersection among the finite amplitude curves; thereby, transitions will be identified. In addition, LTNE produces both retarding and accelerating effects on nonlinear frequency correction. Both retarding and accelerating effects on heat transfer are observed due to LTNE effects. The region of enhancement of heat flux for oscillatory convection in the smaller $\delta$-domain with increasing strength of rotation increases with an increase in the value of LTNE parameters.

Acknowledgement The authors wish to thank the reviewers and the associate editor for their useful comments which helped in improving the paper considerably.

\section{References}

Banu, N., Rees, D.A.S.: Onset of Darcy-Bénard convection using a thermal non equilibrium model. Int. J. Heat Mass Transf. 45, 2221-2228 (2002)

Barletta, A., Rees, D.A.S.: Local thermal non-equilibrium effects in the Darcy-Bénard instability with isoflux boundary conditions. Int. J. Heat Mass Transf. 55, 384-394 (2012) 
Barletta, A., Rees, D.A.S.: Local thermal non-equilibrium analysis of the thermo-convective instability in an inclined porous layer. Int. J. Heat Mass Transf. 83, 327-336 (2015)

Capone, F., Rionero, S.: Inertia effect on the onset of convection in rotating porous layers via the auxiliary system method. Int. J. Non Linear Mech. 57, 192-200 (2013)

Capone, F., Gentile, M.: Sharp stability results in LTNE rotating anisotropic porous layer. Int. J. Therm. Sci. 134, 661-664 (2018)

Celli, M., Barletta, A., Storesletten, L.: Local thermal non-equilibrium effects in the Darcy-Bénard instability of a porous layer heated from below by a uniform flux. Int. J. Heat Mass Transf. 67, 902-912 (2013)

Falsaperla, P., Mulone, G., Straughan, B.: Rotating porous convection with prescribed heat flux. Int. J. Eng. Sci. 48, 685-692 (2010)

Govender, S.: Coriolis effect on the linear stability of convection in a porous layer placed far away from the axis of rotation. Transp. Porous Med. 51, 315-326 (2003)

Govender, S., Vadasz, P.: The effect of mechanical and thermal anisotropy on the stability of gravity driven convection in rotating porous media in the presence of thermal non-equilibrium. Transp. Porous Med. 69, 55-66 (2007)

Lee, J., Shivakumara, I.S., Mamatha, A.L.: Effect of nonuniform temperature gradients on thermogravitational convection in a porous layer using a thermal nonequilibrium model. J. Porous Media. 14, 659-669 (2011)

Malashetty, M.S., Shivakumara, I.S., Kulkarni, S.: The onset of Lapwood-Brinkman convection using a thermal non-equilibrium model. Int. J. Heat Mass Transf. 48, 1155-1163 (2005a)

Malashetty, M.S., Shivakumara, I.S., Sridhar, K.: The onset of convection in an anisotropic porous layer using a thermal nonequilibrium model. Transp. Porous Med. 60, 199-215 (2005b)

Malashetty, M.S., Swamy, M.: The effect of rotation on the onset of convection in a horizontal anisotropic porous layer. Int. J. Therm. Sci. 46, 1023-1032 (2007)

Malashetty, M.S., Swamy, M.: Effect of rotation on the onset of thermal convection in a sparsely packed porous layer using a thermal non-equilibrium model. Int. J. Heat Mass Transf. 53, 3088-3101 (2010)

Malashetty, M.S., Swamy, M., Kulkarni, S.: Thermal convection in a rotating porous layer using a thermal nonequilibrium model. Phys. Fluids 19, 054102 (2007)

Nield, D.A., Bejan, A.: Convection in Porous Media, pp. 37-55. Springer, Berlin (2017)

Palm, E., Tyvand, P.A.: Thermal convection in a rotating porous layer. J. Appl. Math. Phys. (ZAMP) 35, 122-123 (1984)

Rees, D.A.S., Pop, I.: Local thermal non-equilibrium in porous medium convection. Transp. Porous Med. 3, 147-173 (2005)

Rees, D.A.S.: The onset and nonlinear development of vortex instabilities in a horizontal forced convection boundary layer with uniform surface suction. Transp. Porous Med. 77, 243-265 (2009)

Rees, D.A.S.: Microscopic modelling of the two-temperature model for conduction in heterogeneous media. J. Porous Media. 13, 125-143 (2010)

Saravanan, S.: Centrifugal acceleration induced convection in a magnetic fluid saturated anisotropic rotating porous medium. Transp. Porous Med. 77, 79-86 (2009)

Saravanan, S., Sivakumar, T.: Onset of thermovibrational filtration convection departure from thermal equilibrium. Phys. Rev. E 84, 026307 (2011)

Saravanan, S., Brindha, D.: Onset of centrifugal filtration convection: departure from thermal equilibrium. Proc. R. Soc. 469, 20120655 (2013)

Straughan, B.: A sharp nonlinear stability threshold in rotating porous convection. Proc. R. Soc. 457, 87-93 (2001)

Straughan, B.: Global nonlinear stability in porous convection with a thermal non-equilibrium model. Proc. R. Soc. A. 462, 409-418 (2006)

Straughan, B.: Green-Naghdi fluid with non-thermal equilibrium effects. Proc. R. Soc. A. 466, 2021-2032 (2010)

Straughan, B.: Porous convection with local thermal non-equilibrium effects and Cattaneo effects in the solid. Proc. R. Soc. Lond. A 469, 20130187 (2013)

Straughan, B.: Exchange of stability in Cattaneo-LTNE porous convection. Int. J. Heat Mass Transf. 89, 792-798 (2015)

Vadasz, P.: Fluid flow through heterogeneous porous media in a rotating square channel. Transp. Porous Med. 12, 43-54 (1993a)

Vadasz, P.: Three-dimensional free convection in a long rotating porous box. J. Heat Transf. 115, 639-644 (1993b)

Vadasz, P.: Centrifugally generated free convection in a rotating porous box. Int. J. Heat Mass Transf. 37, 2399-2404 (1994)

Vadasz, P.: Convection and stability in a rotating porous layer with alternating direction of the centrifugal body force. Int. J. Heat Mass Transf. 39, 1639-1647 (1996) 
Vadasz, P.: Coriolis effect on gravity-driven convection in a rotating porous layer heated from below. J. Fluid Mech. 376, 351-375 (1998)

Vadasz, P., Govender, S.: Stability and stationary convection induced by gravity and centrifugal forces in a rotating porous layer distant from the axis of rotation. Int. J. Eng. Sci. 39, 715-732 (2001)

Publisher's Note Springer Nature remains neutral with regard to jurisdictional claims in published maps and institutional affiliations. 